\title{
TMEM16B Calcium-Activated Chloride Channels Regulate Action Potential Firing in Lateral Septum and Aggression in Male Mice
}

\author{
는 Wynn Wang, ${ }^{1,2,4}$ Jeffrey Simms, ${ }^{3}$ Christian J. Peters, ${ }^{2,4,5}$ Marena Tynan-La Fontaine, ${ }^{2,4}$ Kexin Li, $^{2,4}$ T. Michael Gill, ${ }^{3}$ \\ 가 Yuh Nung Jan, ${ }^{2,4}$ and (ㄴ) Lily Y. Jan ${ }^{2,4}$ \\ ${ }^{1}$ Neuroscience Graduate Program, University of California San Francisco, San Francisco, California 94143, 2Department of Physiology, University of \\ California San Francisco, San Francisco, California 94143, ${ }^{3}$ Gladstone Institutes Behavioral Core, San Francisco, California 94158, ${ }^{4}$ Howard Hughes Medical \\ Institute, University of California San Francisco, San Francisco, California 94143, and 5Department of Anatomy and Cell Biology, University of Illinois at \\ Chicago, Chicago, Illinois 60612
}

The lateral septum (LS) plays an important role in regulating aggression. It is well recognized that LS lesions lead to a dramatic increase in aggressive behaviors. A better understanding of LS neurophysiology and its functional output is therefore important to assess LS involvement in regulating aggression. The LS is a heterogeneous structure that maintains inputs and outputs with multiple brain regions, and is also divided into subregions that innervate one another. Thus, it is challenging to identify the exact cell type and projections for characterization. In this study, we determined the expression pattern of the calcium-activated chloride channel, TMEM16B, in the LS of both male and female mice. We then investigated the physiological contribution of the calcium-activated chloride channel to LS neuronal signaling. By performing whole-cell patch-clamp recording, we showed that TMEM16B alters neurotransmitter release at the hippocampal-LS synapse, and regulates spike frequency and spike frequency adaptation in subpopulations of LS neurons. We further demonstrated that loss of TMEM16B function promotes lengthened displays of aggressive behaviors by male mice during the resident intruder paradigm. In conclusion, our findings suggest that TMEM16B function contributes to neuronal excitability in subpopulations of LS neurons and the regulation of aggression in male mice.

Key words: aggression; Ano2; Anoctamin 2; CaCC; lateral septum; TMEM16B

Significance Statement

Aggression is a behavior that arose evolutionarily from the necessity to compete for limited resources and survival. One particular brain region involved in aggression is the lateral septum (LS). In this study, we characterized the expression of the TMEM16B calcium-activated chloride channel in the LS and showed that TMEM16B regulates the action potential firing frequency of LS neurons. We discovered that loss of TMEM16B function lengthens the displays of aggressive behaviors in male mice. These findings suggest that TMEM16B plays an important role in regulating LS neuronal excitability and behaviors associated with LS function, thereby contributing to our understanding of how the LS may regulate aggression.

\section{Introduction}

Transmembrane protein of unknown function 16B (TMEM16B, also known as Anoctamin-2 or Ano2) is a calcium-activated chlo-

Received Dec. 14, 2018; revised June 11, 2019; accepted July 11, 2019.

Author contributions: L.W., J.S., C.J.P., T.M.G., Y.N.J., and L.Y.J. designed research; L.W., J.S., and M.T.-L.F. performed research; L.W. and J.S. analyzed data; L.W. wrote the first draft of the paper; L.W., J.S., C.J.P., K.L., T.M.G., Y.N.J., and L.Y.J. edited the paper; L.W., C.J.P., and L.Y.J. wrote the paper; K.L. contributed unpublished reagents/ analytic tools.

This work was supported by the National Institutes of Health Grant R01NS069229. Y.N.J. and L.Y.J. are Howard Hughes Medical Institute investigators. We thank Alexandra Nelson, Robert Edwards, and Vikaas Sohal for discussion and guidance; the Gladstone Institute for Disease Behavioral Core for assisting in behavioral experiments; and Eirish Sison for technical support. ride channel ( $\mathrm{CaCC}$ ) important for regulating neuronal signaling throughout the CNS, including the hippocampus (Huang et al., 2012), inferior olive (Zhang et al., 2017), thalamus (Ha et al., 2016), retina (Stöhr et al., 2009), and olfactory epithelium (Pietra et al., 2016). Among these brain regions, TMEM16B regulates EPSP amplitude (Huang et al., 2012), spike duration (Huang et al., 2012), spike frequency (Pietra et al., 2016; Zhang et al., 2017), and/or spike frequency adaptation (Ha et al., 2016). TMEM16B

The authors declare no competing financial interests.

Correspondence should be addressed to Lily Y. Jan at lily.jan@ucsf.edu.

https://doi.org/10.1523/JNEUROSCI.3137-18.2019

Copyright $\odot 2019$ the authors 
activation, either by calcium influx or release of internally stored calcium, leads to chloride currents that help maintain the membrane potential below threshold for spike firing (Pifferi et al., 2009; Stöhr et al., 2009; Stephan et al., 2009; Sagheddu et al., 2010). Since calcium influx typically accompanies or induces neuronal signaling, TMEM16B provides a powerful means of regulating neuronal activity. Understandably, elimination of TMEM16B can lead to behavioral deficits. TMEM16B KO mice display impaired motor learning correlated with changes in inferior olivary neuronal spike frequency (Zhang et al., 2017), and behavioral deficits in odor-guided food finding indicative of $\mathrm{CaCC}$ mediation of olfactory sensory neuron signal amplification (Pietra et al., 2016). Mice with TMEM16B viral knockdown in the thalamus exhibit increased visceral pain responses and decreased spike-firing adaptation of thalamocortical neurons (Ha et al., 2016).

The lateral septum (LS) is a brain region highly implicated in aggression (Spiegel et al., 1940; Brady and Nauta, 1953; Albert and Chew, 1980). LS lesions in mammals lead to dramatic displays of aggression, first coined as "septal rage," and characterized by increased biting, attacks, and defensive behaviors (Spiegel et al., 1940; Brady and Nauta, 1953, 1955). Similar behavioral alterations result from injection of local anesthetics into the LS (Albert and Richmond, 1976; Albert and Wong, 1978). In support of these findings, LS input to the ventromedial hypothalamus $(\mathrm{VMH})$ is responsible for dampening aggression (Brayley and Albert, 1977; Wong et al., 2016). The LS is primarily composed of GABAergic projection neurons (Risold and Swanson, 1996). Of the three subregions that comprise the LS (the caudal LS [LSc], rostral LS [LSr], and ventral LS [LSv]), the VMH receives input primarily from the LSv (Risold and Swanson, 1997; Toth et al., 2010). The hippocampus is a major source of input to the LS (Risold and Swanson, 1997). A recent study identified a neural circuit involving hippocampal CA2 pyramidal neurons in regulating LS input to the $\mathrm{VMH}$, whereby $\mathrm{CA} 2$ innervation of neurons in the dorsal regions of the LS (including the LSc and LSr) leads to LSv inhibition and VMH disinhibition so as to trigger aggression (Leroy et al., 2018). This circuit demonstrates the complexity of LS function in regulating aggression.

A survey of TMEM16B mRNA expression patterns using the Allen Brain Atlas ISH Database localizes TMEM16B expression to the LSc and LSr, but not the LSv. We have validated TMEM16B expression in these LS subregions with immunohistochemical staining of mice with farnesylated mCherry inserted into the TMEM16B locus, which is simultaneously knocked out in the process (Zhang et al., 2017), and these results have led us to speculate that TMEM16B channels may play a functional role in regulating LS neural circuitry and aggressive behaviors.

In this study, we used this TMEM16B mCherry mouse line (Zhang et al., 2017) to determine how CaCC contributes to LS neuronal signaling and aggressive behaviors. We found that TMEM16B KO mice display prolonged aggressive attacks compared with WT control mice. We identified a CaCC in the LS of WT but not KO mice, and showed that TMEM16B regulates spike frequency and spike frequency adaptation in subpopulations of LS neurons. Collectively, these findings suggest that TMEM16B functions to regulate spike firing in LS neurons, and loss of TMEM16B leads to increased spike firing and longer displays of aggression in male mice.

\section{Materials and Methods}

Animals. All experimental mice used in this study were bred from the TMEM16B mCherry KO mouse line (Zhang et al., 2017). All animal experiments were done in strict accordance with the Guide for the care and use of laboratory animals by the National Institutes of Health. All animal protocols used in this study were approved by the Institutional Animal Use and Care Committee at the University of California San Francisco.

Acute brain slice preparation. Male and female TMEM16B WT and KO mice 16-32 weeks old were anesthetized using isoflurane and perfused with ice-cold NMDG-based cutting solution (in $\mathrm{mm}$ ) as follows: 93 NMDG, $2.5 \mathrm{KCl}, 1.2 \mathrm{NaH}_{2} \mathrm{PO}_{4}, 20$ HEPES, 25 glucose, 5 sodium ascorbate, 2 mannitol, 3 sodium pyruvate, $10 \mathrm{MgSO}_{4}, 0.5 \mathrm{CaCl}_{2}, \mathrm{pH} \sim 7.3-7.4$, $\sim 300-310$ mOsm. Brains were immediately removed and submerged in ice-cold NMDG cutting solution. Brains were sliced into $350 \mu \mathrm{m}$ sections using a VT 1000 S vibratome (Leica Microsystems). Slices were placed in a holding chamber in NMDG-based cutting solution at $33^{\circ} \mathrm{C}$ for $15 \mathrm{~min}$, then transferred to a second holding chamber at room temperature containing HEPES-based ACSF solution (in $\mathrm{mm}$ ) as follows: $92 \mathrm{NaCl}, 2.5$ $\mathrm{KCl}, 1.2 \mathrm{NaH}_{2} \mathrm{PO}_{4}, 30 \mathrm{NaHCO}_{3}, 20$ HEPES, 25 glucose, 5 sodium ascorbate, 2 mannitol, 3 sodium pyruvate, $2 \mathrm{MgSO}_{4}, 2 \mathrm{CaCl}_{2}, \mathrm{pH} 7.3-7.4$, $\sim 300-310$ mOsm. All solutions were equilibrated with $5 \% \mathrm{CO}_{2}+95 \%$ $\mathrm{O}_{2}$. Slices were held at room temperature for at least $45 \mathrm{~min}$ before being used for recordings.

Slice electrophysiology and optogenetic recordings. Whole-cell patchclamp recordings were performed on prepared brain slices and continuously perfused with ACSF using a peristaltic pump and equilibrated with $5 \% \mathrm{CO}_{2}+95 \% \mathrm{O}_{2}$. For EPSPs, mEPSCs, and action potential recordings, slices were perfused with ACSF using the following recipe (in $\mathrm{mM}$ ): $127 \mathrm{NaCl}, 1.8 \mathrm{KCl}, 26 \mathrm{NaHCO}_{3}, 12 \mathrm{KH}_{2} \mathrm{PO}_{4}, 1.3 \mathrm{MgSO}_{4}, 2.4 \mathrm{CaCl}_{2}, 15$ glucose. The $\mathrm{pH}$ was adjusted to within a range of 7.3-7.4 using $\mathrm{HCl}$. Osmolarity was adjusted to a range of 300-305 mOsm. A K-methanesulfonate-based internal solution was used (in mM) as follows: 135 K-methanesulfonate, $6.5 \mathrm{KCl}, 2 \mathrm{MgCl}_{2}$, $4 \mathrm{Na}$-ATP, 0.2 EGTA, 10 HEPES, $\mathrm{pH} \sim 7.2$ with $\mathrm{KOH}, \sim 290-300 \mathrm{mOsm}$. The slice chamber was kept at $33^{\circ} \mathrm{C}$ during recordings.

For tail current recordings, slices were perfused with an NMDG-Cl ACSF solution (in mM) as follows: $120 \mathrm{NMDG}, 20 \mathrm{HEPES}, 1.3 \mathrm{MgSO}_{4}$, $2.5 \mathrm{CaCl}_{2}, 5 \mathrm{TEA}-\mathrm{Cl}, 15$ glucose. A CsCl-based internal solution was used (in mM) as follows: $135 \mathrm{CsCl}, 10 \mathrm{HEPES}, 10 \mathrm{TEA}-\mathrm{Cl}, 2 \mathrm{MgCl}_{2}, 0.1 \mathrm{EGTA}$, $\mathrm{pH} \sim 7.2$ with $\mathrm{Cs} \mathrm{OH}, \sim 290-300 \mathrm{mOsm}$. The slice chamber was kept at room temperature during recordings.

All recordings were done using the patch-clamp amplifier, MultiClamp700B (Molecular Devices), the data acquisition system Digidata 1322A (Molecular Devices), Axioskop2 FS plus upright microscope (Carl Zeiss), and Clampex9 (Molecular Devices). Neurons were visualized with a CCD camera (Hamamatsu). Pipette tips were pulled the day of use with a P-1000 Pipette Puller (Sutter Instruments) with seal resistances of 4-9 $\mathrm{M} \Omega$. Pipette tips were made of thick/standard wall borosilicate glass (ID $0.86 \mathrm{~mm}$, OD $1.50 \mathrm{~mm}$ ). Optogenetic recordings were done using a Lambda DG4 light source (Sutter Instruments). Neutral density filters were used to regulate light intensity and determine the threshold amount of light necessary for EPSP activation. Pharmacological drugs used include $1 \mu \mathrm{M}$ TTX (Tocris Bioscience), $100 \mu \mathrm{M}$ picrotoxin (PTX; Tocris Bioscience), 1 mм kynurenic acid (Sigma-Aldrich), 5 mм 4-AP (SigmaAldrich), and $10 \mathrm{~mm}$ TEA-Cl (Sigma-Aldrich). All drugs were dissolved in the ACSF solution. All electrophysiology data were analyzed using Clampfit10 and Prism 6 (GraphPad, RRID:SCR_002798). Only cells with access resistance $<35 \mathrm{~m} \Omega$ were analyzed. Passive leak was subtracted from tail current recordings offline before subsequent analysis.

Immunohistochemistry. Male and female TMEM16B KO mice 16-32 weeks old were anesthetized using isoflurane and perfused with $1 \times \mathrm{PBS}$, followed by $4 \%$ PFA in $1 \times$ PBS. After brain dissection, brains were placed in $4 \%$ PFA for $4 \mathrm{~h}$ followed by $30 \%$ sucrose for $2 \mathrm{~d}$. Brains were frozen in OCT and sectioned into $30-\mu \mathrm{m}$-thick coronal sections using a CM3050 S cryostat (Leica Microsystems). Brain sections not used immediately were stored in cryoprotectant solution at $-20^{\circ} \mathrm{C}(40 \% 1 \times \mathrm{PBS}$, $30 \%$ ethylene glycol, $30 \%$ glycerol).

Brain sections underwent free-floating immunohistochemistry for antibody labeling. Sections were washed in $1 \times$ PBS for 5 min and then placed in boiling citrate buffer solution $(0.05 \%$ Tween $20,114 \mathrm{~mm}$ sodium citrate, $\mathrm{pH}$ 6.0) for $5 \mathrm{~min}$ to perform antigen retrieval. Following 
two 3 min $1 \times$ PBS washes for cool-down, sections were incubated in blocking solution $(0.3 \%$ Triton, $3 \%$ normal goat serum in $1 \times$ PBS) for $1 \mathrm{~h}$ at room temperature. Sections were incubated in primary antibodies diluted with blocking solution overnight at $4^{\circ} \mathrm{C}$. Primary antibodies used include mouse monoclonal anti-NeuN 1:500 (Millipore, catalog \#MAB377, RRID:AB_2298772), rabbit polyclonal anti-DsRed-Express 1:2500 (Clontech Laboratories, catalog \#632496, RRID:AB_10013483), rabbit polyclonal anti-GFAP 1:100 (Sigma-Aldrich, catalog \#G9269, RRID:AB_477035), rabbit polyclonal anti-Iba1 1:1500 (Wako, catalog \#019-19741, RRID:AB_839504), and chicken polyclonal anti-mCherry 1:4000 (Novus Biologicals, catalog \#NBP2-25158, RRID:AB_2636881). Sections underwent three $10 \mathrm{~min} 1 \times$ PBS washes. Secondary antibodies diluted with blocking solution were applied to sections for $1 \mathrm{~h}$ at room temperature. Secondary antibodies include goat anti-mouse $\operatorname{IgG}(\mathrm{H}+\mathrm{L})$ Alexa-488 1:1000 (Thermo Fisher Scientific, catalog \#A-11001, RRID: AB_2534069), goat anti-rabbit IgG $(\mathrm{H}+\mathrm{L})$ Alexa-568 1:1000 (Invitrogen, catalog \#A-11036, RRID:AB_143011), goat anti-rabbit IgG $(\mathrm{H}+\mathrm{L})$ Alexa-488 1:1000 (Invitrogen, catalog \#A-11008, RRID:AB_143165), and goat anti-chicken IgY\# (IgG) $(\mathrm{H}+\mathrm{L})$ Cy3 1:1000 (Jackson ImmunoResearch Laboratories, catalog \#103-165-155, RRID:AB_2337386). Next, sections underwent three $10 \mathrm{~min} 1 \times$ PBS washes. Sections were mounted onto Superfrost Plus Microscope Slides (Thermo Fisher Scientific). Glass coverslips were placed on top of the slides using $150 \mu$ l DAPI Fluoromount-G mounting media (Southern Biotechnology).

Microscopy and cell count analysis. All fluorescent imaging was done using an SP8 confocal microscope (Leica Microsystems). Tile scan images were taken at $20 \times$ magnification. LS cell count images were taken at $63 \times$ magnification. The LS was divided into 6 or 7 equal areas based on the location of mCherry expression. $z$-stack confocal images with $3 \mu \mathrm{m}$ $z$-step sizes were taken of each LS area, and cells double-labeled for mCherry and the specific cell type marker were manually counted throughout the entire stack using ImageJ (ImageJ, RRID:SCR_003070). The number of double-labeled cells was summed up across all areas of the LS from both brain hemispheres. The sample size for the cell count analysis was $n=10$. For each sample, two $30 \mu \mathrm{m}$ sections were selected for cell count analysis. For quantification of cholera toxin subunit $\mathrm{B}^{+}$ (CTB) cells, tile scan images were taken at $20 \times$ magnification. CTB cells in the CA3 region were manually counted using ImageJ (ImageJ, RRID: SCR_003070).

Behavioral assays. Male TMEM16B WT and KO mice 20-33 weeks of age were transferred to the Gladstone Behavioral Core and acclimated to new housing at least 2 weeks before any testing. Researchers conducting all behavioral tests were blinded to the mouse genotype during testing and analysis. Statistical analysis for all behavioral tests was completed using Prism 6 (GraphPad, RRID:SCR_002798).

Resident intruder paradigm. Resident mice included male TMEM16B WT and KO mice. C57BL6 male mice 16 weeks of age were purchased from The Jackson Laboratory (https://www.jax.org/strain/000664) and served as intruder mice. All intruder mice were group-housed 5 per cage and identified with ear punch(es). Resident mice were identified with a tattoo on their hindpaw toes.

All mice were brought to the testing room and allowed to acclimate $1 \mathrm{~h}$ before testing. For 3 consecutive days, a novel intruder mouse was placed into the home cage $(10$ inch $\times 7$ inch $\times 6.5$ inch $)$ of the resident mouse for a maximum of $10 \mathrm{~min}$. Each intruder mouse was tested only once per day, and each resident mouse was introduced to a different intruder mouse each day. The cohort was split in half and tested over the span of 2 weeks. The resident-intruder social interaction was video recorded for later analysis. Videos were analyzed for the number and duration of "attacks" exhibited by the resident mouse. An "attack" was defined as any moment when a resident mouse was seen biting the intruder mouse. Any attack lasting $\geq 30 \mathrm{~s}$ was immediately terminated, and the remainder of the testing session for that resident was also terminated. For statistical analysis, resident mice that exhibited these types of prolonged "attacks" were given a maximum value equal to the third quartile +1.5 (interquartile range) for the calculated measurements of total time and numbers of attacks based on the genotype-specific population values. This equation was based on the assumption that these mice would be considered outliers in a normally distributed sample set.
Direct social interaction test. Mice were transferred to the testing room $1 \mathrm{~h}$ earlier for acclimation. One experimental mouse and one social stimulus mouse were placed into a novel home cage $(10$ inch $\times 7$ inch $\times 6.5$ inch) absent of any bedding and allowed to directly interact with one another for 10 min. C57BL6 male mice 16 weeks of age from The Jackson Laboratory (https://www.jax.org/strain/000664) were used as social stimulus mice. Stimulus mice were labeled on their back with an odorless marker to differentiate between the stimulus and experimental mice. Each stimulus mouse was used up to three times per day, each time with a different experimental mouse. Interactions were video recorded and analyzed by hand for the total amount of time mice spent directly interacting with one another. Interactions included perioral contact, anogenital contact, rough play, etc. The novel home cage was cleaned in between mice with $70 \%$ ethanol.

Two chamber social approach test. The two-chamber social approach test measures sociability by determining the amount of time experimental mice spend and interact with a social stimulus mouse on the "social side" versus the "nonsocial side" of the chamber. The test was conducted using white acrylic boxes $(24$ inch $\times 16$ inch $\times 8.75$ inch $)$ that were placed inside larger light and sound attenuating cubicles (Lafayette Instruments). The boxes were split in half using a clear acrylic divider with an archway opening in the bottom middle that allowed access between the two sides of the box. The upper left and right corners of each chamber contained two small enclosures, one of which housed the social stimulus mouse. C57BL6 male mice 16 weeks of age from The Jackson Laboratory (https://www.jax.org/strain/000664) were used as the social stimulus mice.

Mice were transferred to the testing room $1 \mathrm{~h}$ before start of testing. Before social approach testing, the social stimulus mice were habituated to the enclosure space for two 15 min sessions. Between sessions, the stimulus mice were returned to their home cage for an $\sim 20$ min intertrial interval. On the day of social approach testing, the experimental mice first underwent a $10 \mathrm{~min}$ habituation trial, where they were allowed to freely explore the chambers and empty enclosures. Next, the experimental mouse was blocked on the nonsocial side of the chamber while the social stimulus mouse was placed into the small enclosure on the "social side." The social approach trial was then immediately conducted with the experimental mouse again being allowed to freely explore the chambers and enclosures for an additional $10 \mathrm{~min}$. Chambers were cleaned with $70 \%$ ethanol between each experimental mouse tested. All trials were video recorded with cameras mounted from above. Videos were later analyzed using TopScan software (CleverSys) for the total number of bouts and time spent on the "social" and "nonsocial" sides of the chamber, and within a $2 \mathrm{~cm}$ "interaction" zone around the "social" and "nonsocial" enclosures.

Morris Water Maze (MWM) cued platform training test. Mice underwent testing using the cued platform version of the MWM task. The training was conducted using a pool $(122 \mathrm{~cm}$ diameter, $50 \mathrm{~cm}$ high $)$ filled with opaque water with the addition of white, nontoxic tempera paint to test visual procedural learning ability. The testing consisted of two phases: pretraining and cued platform testing. The pretraining phase took place on day 1 in which the mice learned to swim to a hidden platform submerged under the water in the middle of a rectangular training track $(15 \mathrm{~cm} \times 122 \mathrm{~cm})$ that spanned the length of the pool. Once mice located the platform, they were allowed to remain there for $10 \mathrm{~s}$ before being removed by the experimenter. Mice that jumped off the platform were guided back by the experimenter's hand. Mice underwent 10 consecutive trials, with each successive trial starting from the opposite end of the track during the pretraining phase.

Next, the cued platform training took place on days 2-4. During each trial, mice were placed into the pool facing the wall and allowed $60 \mathrm{~s}$ to find the cued platform, which was submerged $1.5 \mathrm{~cm}$ underwater but visually "cued" by the black-and-white striped mast $(15 \mathrm{~cm}$ tall $)$ placed in its center. Mice were allowed to remain on the cued platform for an additional $10-20 \mathrm{~s}$ before being removed from the pool. The drop location was consistent for all animals but varied from trial to trial, whereas the cued platform location was consistent for all mice but varied per session among the four different pool quadrants. Each testing session consisted of two trials, with a 15 min intertrial interval, and two sessions 
were conducted each day with an $\sim 3 \mathrm{~h}$ intersession interval. All sessions were recorded and measurements were made with EthoVision video tracking system (Noldus Information Technology).

Visual placing response. The visual placing response of each mouse was determined by suspending them by the tail and lowering them toward a solid surface, such as a countertop. If the mouse lifted its head and reached its forelimbs toward the object, it was considered to have a normal visual placing response.

Stereotaxic surgery. Mice were anesthetized with $2 \%$ vaporized isoflurane with $\mathrm{O}_{2}$ and administered a mixture of ketamine $(80-100 \mathrm{mg} / \mathrm{kg}$ ) and xylazine $(5-10 \mathrm{mg} / \mathrm{kg})$ via intraperitoneal injection and subcutaneous injections of meloxicam (5-10 mg/kg), buprenorphine (0.05-01 mg/ $\mathrm{kg}$ ), and ampicillin (10-20 mg/kg). Mice were prepped for surgery by shaving and cleaning the head and skull with alcohol wipes and iodine. Surgeries were done using a Robot Stereotax (Neurostar Stereotaxic), SZ61 microscope (Olympus), and a constant flow of $1 \%$ vaporized isoflurane with $\mathrm{O}_{2}$. Mice were injected using a Hamilton syringe with $500 \mathrm{nl}$ of AAV5-CaMKII-hChR2(H134R)-EYFP with titer $\geq 1 \times 10^{13} \mathrm{vg} / \mathrm{ml}$ (Penn Vector Core, Deisseroth Laboratory). Coordinates used for experiments include the following: CA3 region $(-2.18 \mathrm{AP},-2.14 \mathrm{ML}, 2.21$ DV) and the lateral hypothalamic area (LHA; $-1.46 \mathrm{AP},-0.85 \mathrm{ML}, 5.1$ DV). For retrograde tracing studies, mice were injected with $500 \mathrm{nl}$ of CTB (Invitrogen) into the LS (0.14 AP, $-0.22 \mathrm{ML}, 2.6 \mathrm{DV})$.

On postsurgical day 1 , mice were given subcutaneous injections of meloxicam $(5-10 \mathrm{mg} / \mathrm{kg})$ and ampicillin $(10-20 \mathrm{mg} / \mathrm{kg})$. Mice were also given subcutaneous injections of buprenorphine $(0.05-01 \mathrm{mg} / \mathrm{kg})$ if pain was observed. Mice were monitored $4 \mathrm{~d}$ after surgery, and weight measurements were recorded. At 17-21 d after surgery, mice were prepped for whole-cell patch-clamp physiology experiments according to the "acute slice preparation" section.

Experimental design and statistical analysis. This study used TMEM16B WT and KO mice bred from the TMEM16B mCherry KO mouse line (Zhang et al., 2017). All data were analyzed using Prism 6 (GraphPad), and all values reported are mean \pm SEM. For electrophysiology data, a repeated-measures two-way ANOVA statistical analysis with multiple comparisons was conducted for statistical analysis of EPSP, tail current, and spike-firing data. The EPSP data site used a Tukey's multiplecomparison test, whereas the tail current and spike-firing data used a Sidak's multiple-comparison test with an $\alpha$ value of $p<0.05$. Multiple Student's $t$ test analysis was used for input-output curves generated from the LS neuron subpopulation dataset with an $\alpha$ value of $p<0.05$. Student's $t$ test was conducted for mEPSC and EPSP paired pulse analysis with an $\alpha$ value of $p<0.05$. For cell count data, the column statistics were calculated to determine the mean \pm SEM of the percentage of doublelabeled cells. Student's $t$ test analysis was conducted for the CTB ${ }^{+}$retrograde tracing experiment with an $\alpha$ value of $p<0.05$. For the resident intruder paradigm, a repeated-measures two-way ANOVA statistical analysis with Sidak's multiple-comparisons test was conducted when analyzing all mice in the sample size. Welch's $t$ test analysis was conducted when analyzing the time/attack for the subsets of mice that actually attacked each day of the paradigm. For the two-chamber social approach test, a repeated-measures two-way ANOVA statistical analysis with Sidak's multiple-comparisons test was conducted. An $\alpha$ value of $p<$ 0.05 was used for detecting statistical significance. Welch's $t$ test analyses were conducted for Direct Social Interaction Test and MWM cued platform training with an $\alpha$ value of $p<0.05$. For the MWM, a percentage rank was calculated for measurements of each mouse for latency, distance, and swim speed. Percentage rank values were averaged across all sessions of the test. The average percentage rank for each mouse was then used for Welch's $t$ test analysis.

\section{Results}

\section{TMEM16B is expressed in the mouse LS}

To examine the expression of TMEM16B in the LS, we used a TMEM16B KO mouse line generated in-house (Zhang et al., 2017), wherein a farnesylated mCherry marker followed by a stop codon is expressed in-frame at exon 3. This results in expression of mCherry that is associated with the cell membrane in cells normally expressing TMEM16B in heterozygous control mice, as well as homozygous mice with loss (KO) of TMEM16B function. Using this mouse line, we identified mCherry expression in the LS in a series of slices along the anterior-posterior axis (Fig. 1A), and observed expression in the LSc and LSr, but not the LSv. Furthermore, we double-labeled TMEM16B KO brain sections with mCherry and markers for specific cell types, including neurons with NeuN (Fig. 1B), astrocytes with GFAP (Fig. 1C), and microglia with Ibal (Fig. 1D). Quantification of $\mathrm{NeuN}^{+}$cells colabeled with mCherry indicates that the great majority $(95.50 \pm 0.44 \%$; mean \pm SEM; $n=10)$ of LS neurons are TMEM16B-expressing neurons (Fig. 1E). Double-labeling of mCherry with GFAP and Iba1 showed little overlap, with $2.65 \pm$ $0.49 \%$ (mean \pm SEM; $n=10$ ) of LS astrocytes expressing mCherry and $1.34 \pm 0.51 \%$ (mean \pm SEM; $n=10$ ) of LS microglial cells expressing mCherry (Fig. 1E), suggesting that TMEM16B expression is primarily restricted to LS neurons. In addition, we found levels of TMEM16B expression in these specific cell types of the LS to be comparable between male $(n=5)$ and female $(n=5)$ mice (Fig. $1 F$; Student's $t$ test; mCherry ${ }^{+}$/ $\mathrm{NeuN}^{+}$cells, $p=0.8809 ; \mathrm{mCherry}^{+} / \mathrm{GFAP}^{+}$cells, $p=0.4016$; mCherry $^{+} / \mathrm{Ibal}^{+}$cells, $\left.p=0.2039\right)$. Based on these results, we conclude that TMEM16B is expressed in neurons in subregions of the LS.

We were also able to determine the output regions of TMEM16B-expressing LS neurons based on the farnesylated mCherry expression. We identified mCherry nerve fibers in certain brain regions, including the lateral preoptic area (LPO) and LHA (Fig. 2). Since no TMEM16B mRNA expression was seen in these regions, the mCherry expression we observed could be attributed to LS neuronal projections that express TMEM16B. Neuronal tracing studies have identified both LSc and LSr input to both the LPO and LHA (Risold and Swanson, 1997), consistent with the notion that TMEM16B-expressing LS neurons project to these areas of the hypothalamus. We also found mCherry nerve fibers in LSv (Fig. 2D), indicating that LSv neurons without TMEM16B expression receive input from TMEM16B expressing LS neurons.

\section{TMEM16B KO mice spend more time engaged in aggressive behavior}

Given the robust expression of TMEM16B in LS neurons, we hypothesized that loss of TMEM16B function may lead to LSrelated behavioral deficits. Studies examining LS function have identified its role in regulating aggression (Brady and Nauta, 1953; Albert and Chew, 1980; Wong et al., 2016; Leroy et al., 2018). Based on this rationale, we speculated that TMEM16B expression in the LS is indicative of potential involvement in aggression. To test this hypothesis, we performed a series of behavioral tests designed to examine aggression behaviors in TMEM16B KO mice and compared these mutants with WT littermate controls to look for behavioral phenotypes (Figs. 3, 4).

Mouse aggression has typically been studied in male mice because male mice are known to fight more than female mice (Miczek et al., 2001). Hence, we generated an initial cohort of male adult TMEM16B WT mice $(n=17)$ and KO mice $(n=15)$. We performed the resident intruder paradigm to examine aggressive behavior in TMEM16B KO mice and WT littermates (Fig. $3 A)$. We observed striking trends in the dataset, suggesting that TMEM16B KO mice exhibited longer displays of aggression compared with WT, although the differences did not reach statistical significance. To determine the reproducibility of these results, we generated a second cohort of adult male TMEM16B WT mice 


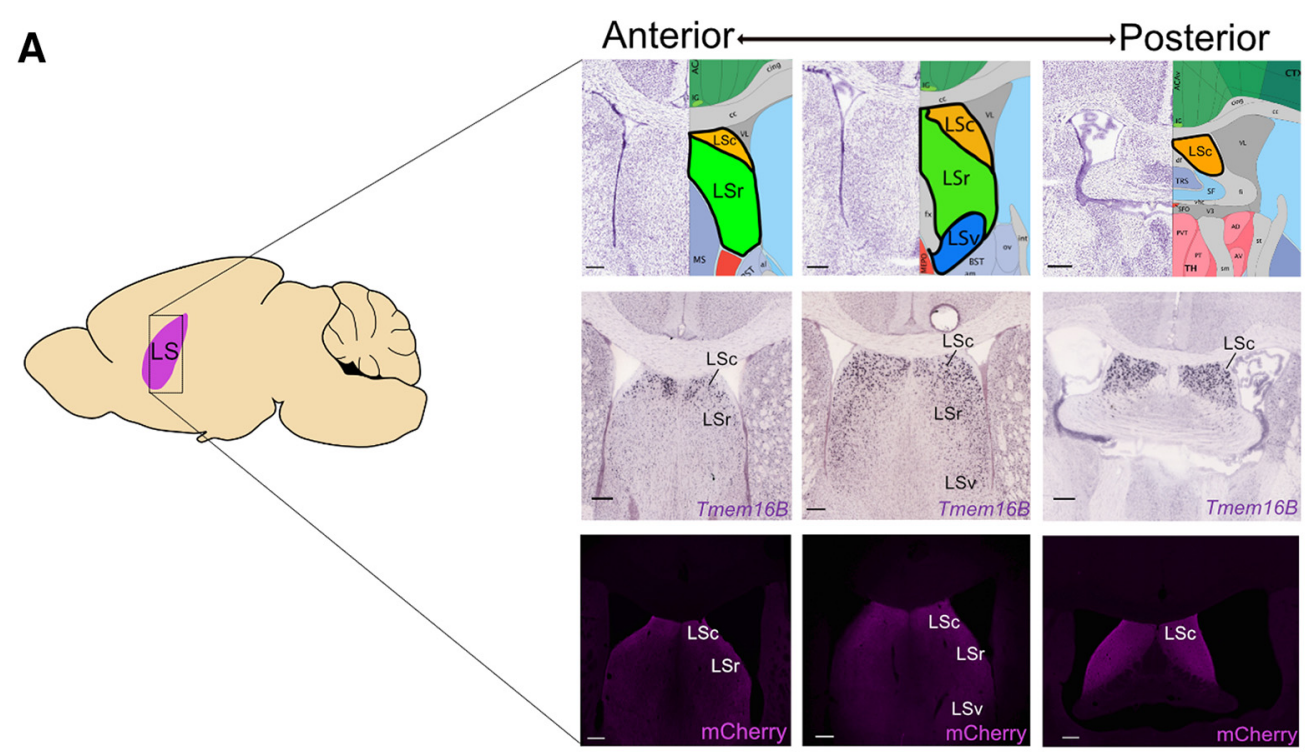

B
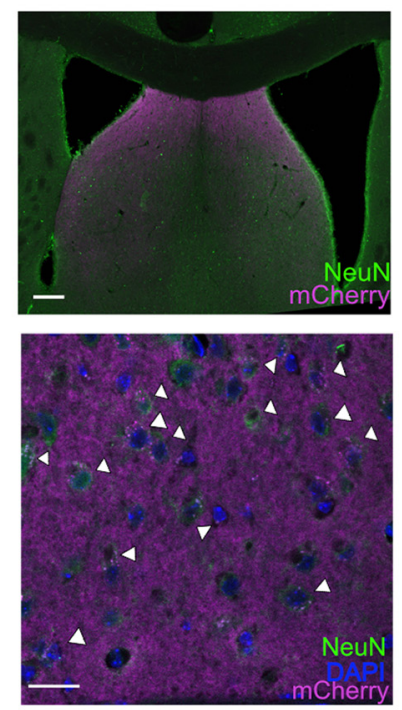

E

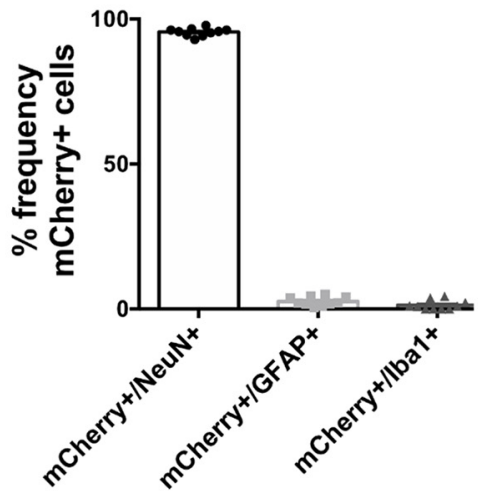

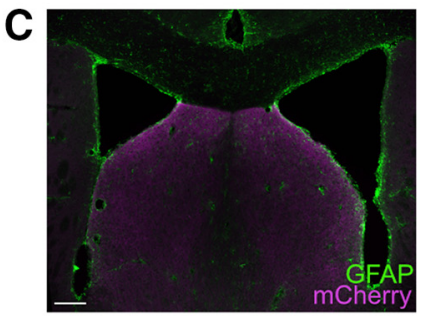

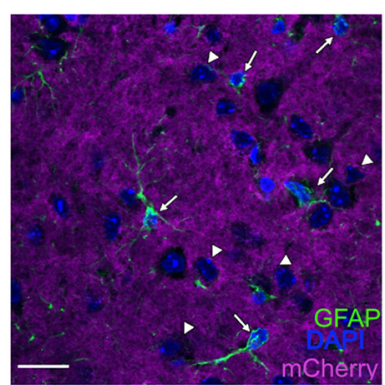

$\mathbf{F}$

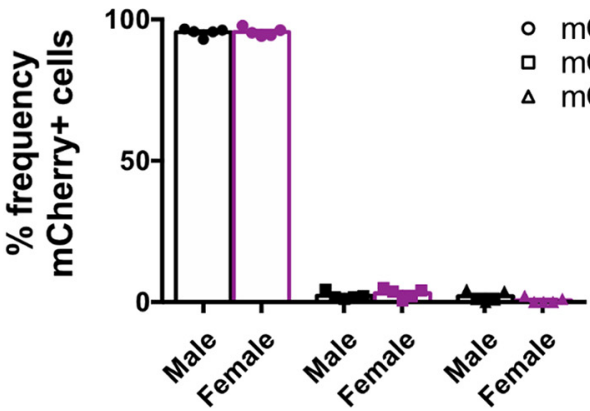

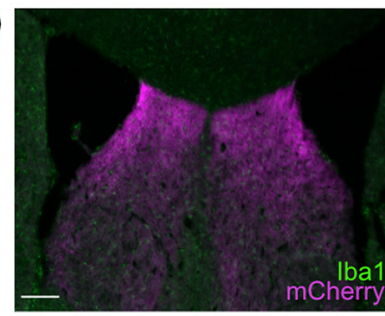

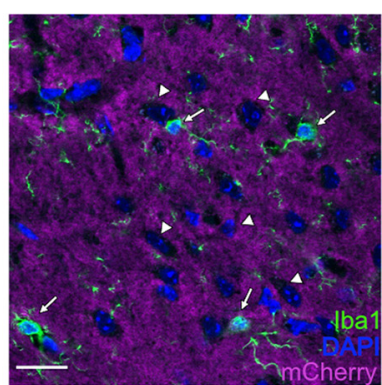

- mCherry+/NeuN+

mCherry+/GFAP+

mCherry+/lba1+

Figure 1. TMEM16B is expressed in $L S$ neurons. $A$, Schematic of sagittal mouse section with location of $L S$ (left), with depiction of $L S$ subregion locations (top right), TMEM16B mRNA (middle right), and TMEM16B protein (bottom right) expression from the anterior to posterior end of the LS. LS subregion images and in situ hybridization images of TMEM16B (gene name: Ano2) in WT mice are modified from the Allen Brain Reference Atlas and Allen Brain ISH database (experiment \#71587834), respectively. Fluorescence images are from TMEM16B K0 mice with a simultaneous in-frame knock in of a farnesylated mCherry marker. LS subregions include LSc, LSr, and LSv. Sagittal mouse brain diagram credit: Jonas Töle, Creative Commons License. $B$, Fluorescent double labeling for NeuN and mCherry in TMEM16B K0 LS sections shows mCherry expression along the membrane of TMEM16B-expressing neurons. Top, Tile scan 20× image with stitching. Bottom, 63X immunohistochemistry image for NeuN and mCherry shows overlapping expression (arrowheads) of mCherry in NeuN ${ }^{+}$cells. $\boldsymbol{C}, \boldsymbol{D}$, Fluorescent double labeling for astrocyte marker, GFAP (C), or microglia marker, Iba1 (D) with mCherry in the LS. Top, Tile scan images $20 \times$ with stitching. Bottom, Representative $63 \times$ images. Arrowheads point to $\mathrm{mCherry}^{+}, \mathrm{DAPI}^{+}, \mathrm{GFAP}^{-}$cells $(\boldsymbol{C})$ or mCherry ${ }^{+}, \mathrm{DAPI}^{+}, \mathrm{Iba}^{-}{ }^{-}$cells (D). Arrows point to mCherry ${ }^{-}, \mathrm{DAPI}^{+}, \mathrm{GFAP}^{+}$cells $(\boldsymbol{C})$ or mCherry ${ }^{-}, \mathrm{DAPI}^{+}, \mathrm{Iba}^{+}{ }^{+}$cells (D). DAPI staining was used to identify cell bodies of GFAP ${ }^{+}$and Iba ${ }^{+}$ cells. $\boldsymbol{E}$, Dot plot bar graph of cell count analysis. $\boldsymbol{F}$, Dot plot bar graph analysis of male-to-female comparison. Student's $t$ test analysis shows no significant differences between males and females. Error bars indicate SEM. Sample size: All TMEM16B KO mice, male $n=5$, female $n=5$. For all images: Scale bars: Tile scan image, $200 \mu \mathrm{m} ; 63 \times$ image, $20 \mu \mathrm{m}$. 
A

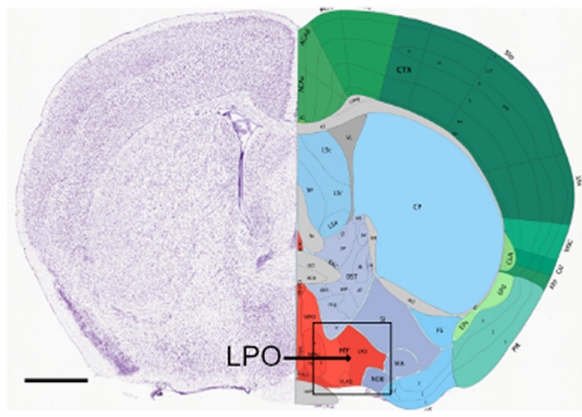

B

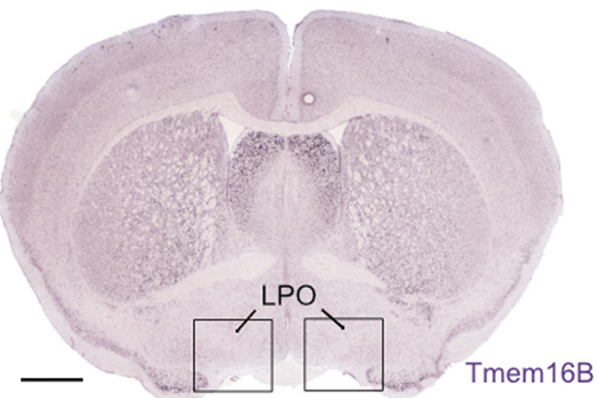

C

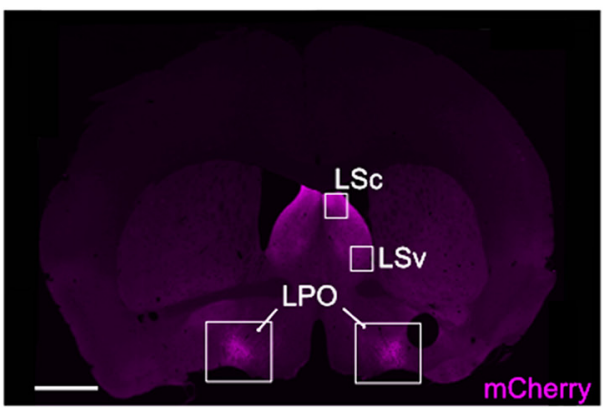

D

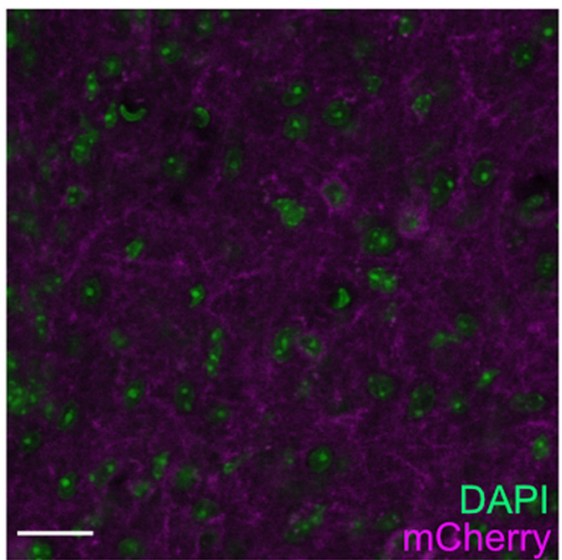

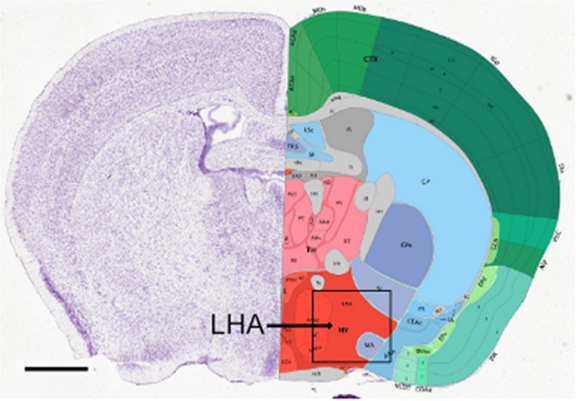
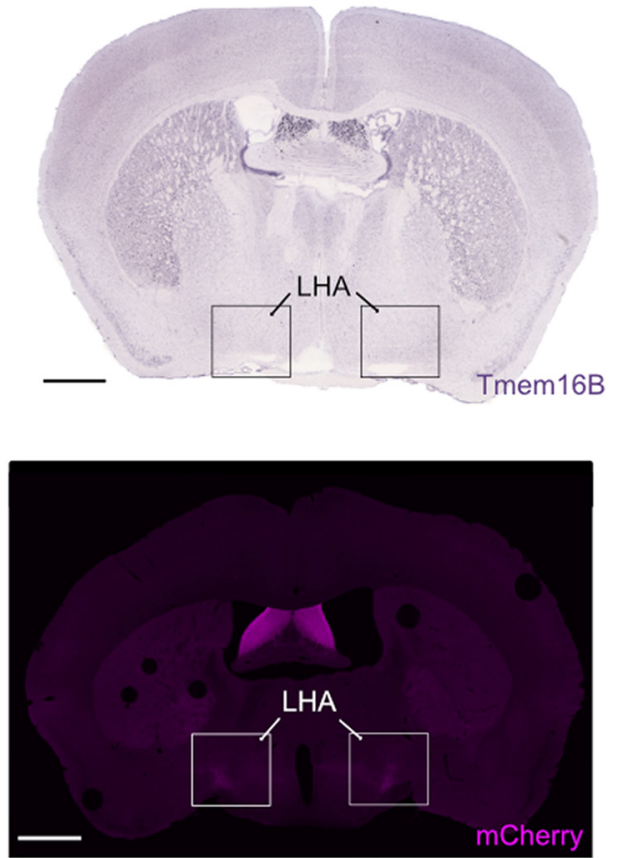

E

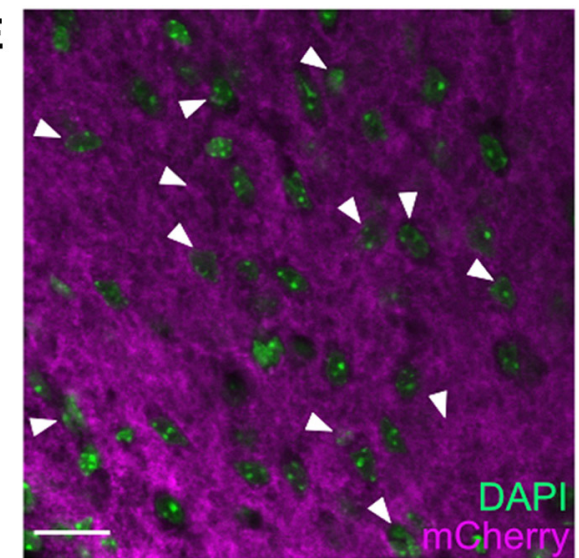

Figure 2. LS neurons with TMEM16B expression project to ventral LS and hypothalamic nuclei. TMEM16B-expressing LS neurons project to LP0 and LHA. $A$, Modified Allen Brain Reference Atlas images with locations of LPO (left) and LHA (right) delineated by black border. B, Modified Allen Brain ISH images for TMEM16B mRNA (gene name: Ano2; experiment \#71587834) (right). Black border indicates absence of TMEM16B mRNA expression in LPO (left) and LHA (right), suggesting that mCherry expression is from LS nerve fibers projecting to these hypothalamic regions. Arrows point to the specific brain region. C, mCherry fluorescence images in coronal section of TMEM16B KO mouse brain. Large white border indicates location of mCherry axon fibers in LPO (left) and LHA (right). Small white borders indicate the caudal $L S(L S c)$, which is part of the dorsal regions of the $L S$, and the $L S v$. Arrows point to the specific brain region. $D, 63 \times$ magnification image of the $L S v$ with DAPI and mCherry labeling. mCherry labels the nerve fibers in the LSv but not the cell membranes surrounding DAPI-labeled cell bodies. E, $63 \times$ magnification image of the LSc with DAPI and $\mathrm{mCherry}$ labeling. mCherry puncta localized to the cell membranes surrounding DAPI-labeled cell bodies (see arrowheads). Scale bars: $63 \times$ images, $20 \mu \mathrm{m}$; all coronal section images, $1000 \mu \mathrm{m}$.

$(n=19)$ and KO mice $(n=20)$ for the resident intruder paradigm. We observed consistent findings across both cohorts: mean total attack time between TMEM16B WT and KO mice was $7.7 \pm$ $3.18 \mathrm{~s}$ and $19.9 \pm 7.03 \mathrm{~s}$ for cohort 1 and $7.9 \pm 4.5 \mathrm{~s}$ and $22.09 \pm$
$9.1 \mathrm{~s}$ for cohort 2, respectively, on day 2 of the paradigm, and $7.7 \pm 3.62 \mathrm{~s}$ and $23.9 \pm 6.6 \mathrm{~s}$ for cohort 1 and $10.29 \pm 4.96 \mathrm{~s}$ and $26.63 \pm 9.9 \mathrm{~s}$ for cohort 2 , respectively, on day 3 of the paradigm. Moreover, our combined results from both cohorts showed a 
A

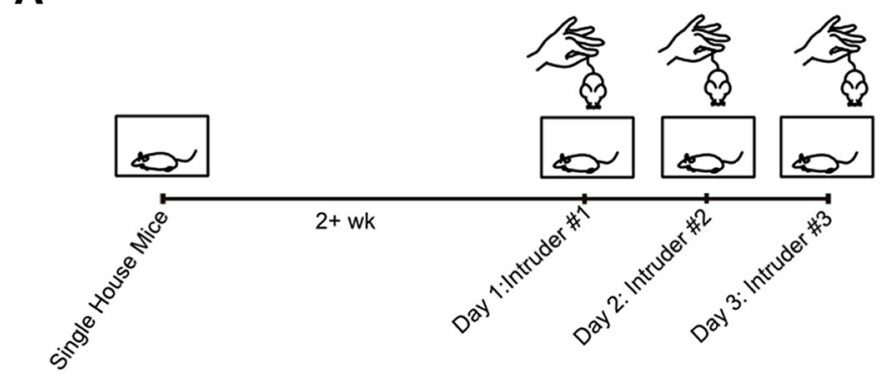

C

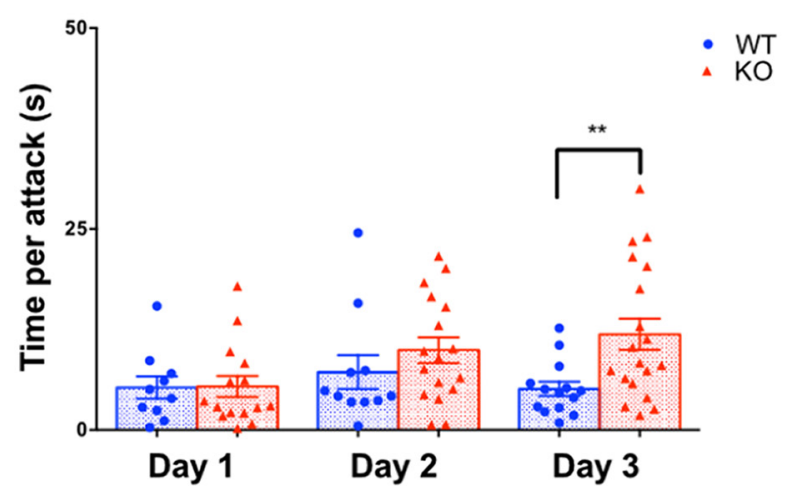

B

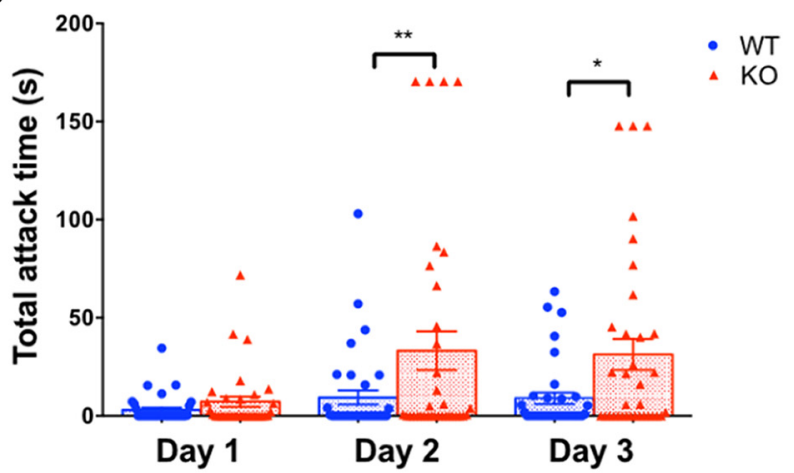

D

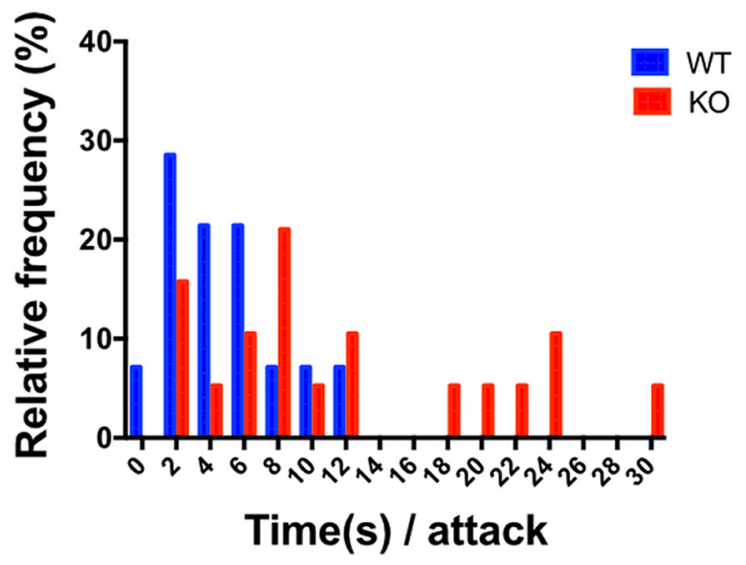

Figure 3. TMEM16B KO mice display longer bouts of aggression compared with WT littermates. $A$, Experimental timeline for resident-intruder paradigm. Experimental mice were introduced to a different intruder mouse each day of testing. B, TMEM16B KO mice display increased total attack time compared with WT littermates on day $2(p=0.0081)$ and day $3(p=0.0141)$. Tw0-way ANOVA with Sidak's multiple comparisons. Data are mean \pm SEM. Sample size: WT, $n=35 ; \mathrm{KO}, n=34$. C, Of the mice that do attack during testing, TMEM16B KO mice display increased mean time per attack compared with WT littermates on day 3 (two-way ANOVA with Sidak's multiple comparisons, $p=0.0037$ ). Data are mean \pm SEM. $\boldsymbol{D}$, Frequency distribution of time/attack for mice that displayed attacks. TMEM16B KO mice display higher median time/attack (8.33 s) compared with WT littermates (4.75 s). Sample size: WT, $n=14 ; \mathrm{KO}, n=19$. Error bars indicate SEM. ${ }^{*} p<0.05$, ${ }^{* *} p<0.01$.

significant increase in the mean amount of time taken by TMEM16B KO mice in attacking the intruder, compared with WT mice on days 2 and 3 (Fig. $3 B$; repeated-measures two-way ANOVA; sources of variation: day, $F_{(2,136)}=4.860, p=0.0091$; genotype, $F_{(1,68)}=8.428, p=0.005$; interaction, $F_{(2,136)}=2.853$, $p=0.0611$; Sidak's multiple comparison: day $1, p=0.9347$; day $2, p=0.0081$; and day $3, p=0.0141$ ). The spread of individual points in Figure $3 B$ revealed that both control and TMEM16B KO mice showed a range of attack times, including examples of resident mice that did not attack. When we only compared the resident mice that did attack each day of testing (WT, $n=14 ; \mathrm{KO}$, $n=19$ ), we observed longer displays of attacks initiated by TMEM16B KO mice compared with WT mice on day 3 of the paradigm, including an increase in the mean time per attack on day 3 (Fig. $3 C$; Welch's $t$ test; day $1, p=0.9479$; day 2, $p=0.3134$; and day $3, p=0.0037$ ) and a rightward shift in the frequency distribution for time per attack on day 3 (Fig. 3D). Collectively, these findings revealed that loss of TMEM16B function in mice increased the duration of aggressive displays toward intruders.

\section{Loss of TMEM16B function in male mice does not affect behaviors associated with social interaction or visual cued learning}

To determine whether our findings from the resident intruder paradigm were influenced by differences in nonaggressive social interaction behavior, we performed additional behavioral tests on the first cohort (Fig. 4). Because we did not observe any trends in behavioral differences between TMEM16B WT and KO mice for these tests, we did not repeat them with the second cohort.

To determine whether differences in sociability between TMEM16B KO and WT mice contributed to our observed aggression phenotype, we performed the two-chamber social approach test (Fig. $4 A$; WT, $n=16$; KO, $n=14$ ). During the habituation trial, WT and TMEM16B KO mice showed equal preference for both sides of the chamber. During the social trial, both WT and TMEM16B KO mice showed greater preference for the chamber side with the social stimulus mouse based on the number of interaction bouts, interaction time, and time spent on each side of the chamber (Fig. $4 A$; repeated-measures two-way ANOVA; "interaction bouts": interaction, $F_{(1,28)}=0.5851, p=$ 0.4507; genotype, $F_{(1,28)}=2.957, p=0.0966$; and chamber side, $F_{(1,28)}=105.3, p<0.0001$; Sidak's multiple comparisons: WT, $p<0.0001 ; \mathrm{KO}, p<0.0001$; "interaction time": interaction, $F_{(1,28)}=0.1643, p=0.6883$; genotype, $F_{(1,28)}=0.4747, p=$ 0.4965 ; and chamber side, $F_{(1,28)}=152.2, p<0.0001$; Sidak's multiple comparisons: WT, $p<0.0001$; KO, $p<0.0001$; "chamber time spent": interaction, $F_{(1,28)}=0.5545, p=0.4627$; genotype, $F_{(1,28)}=1.455, p=0.2378$; and chamber side, $F_{(1,28)}=$ 57.52, $p<0.0001$; Sidak's multiple comparisons: WT, $p<$ $0.0001 ; \mathrm{KO}, p<0.0001)$. Together, these findings suggest that loss of TMEM16B in male mice does not affect sociability levels upon encounter with unfamiliar mice. 
A

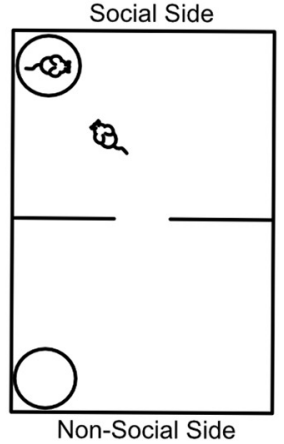

B

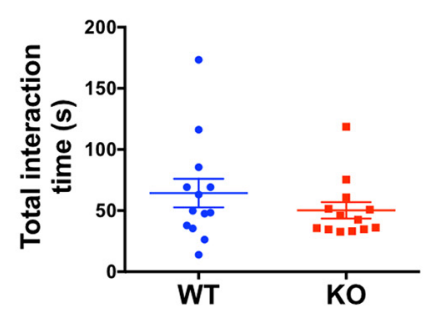

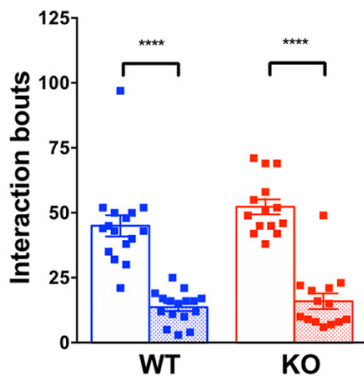

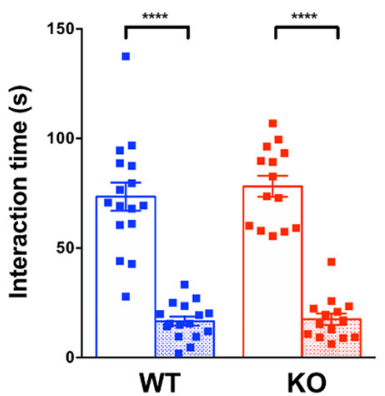

C

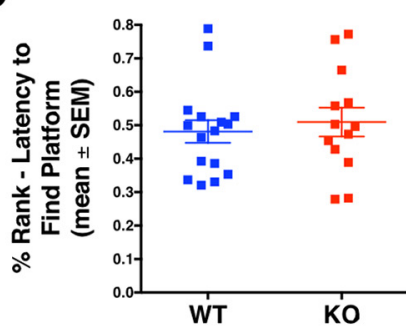

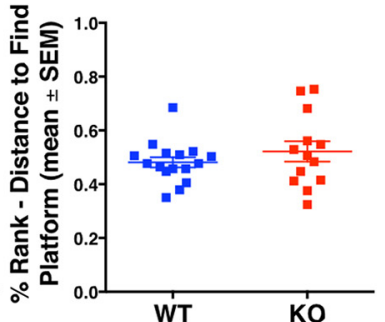

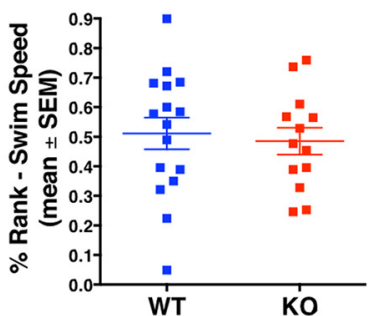

Figure 4. TMEM16B K0 mice show no difference in social interaction behaviors or visual cued learning compared with WT littermates. $A$, Schematic of two chamber social approach test (left). Both TMEM16B WT and K0 mice show a significant preference for the social side of the chamber in terms of interaction bouts (left graph; $p<0.0001$ ), interaction time (middle graph; $p<0.0001)$, and side time (right graph; $p<0.0001$ ). Two-way ANOVA with Sidak's multiple comparisons. Sample size: WT, $n=16 ; \mathrm{KO}, n=14$. B, TMEM16B KO and WT mice show similar levels of interaction time with the stimulus mouse during the direct social interaction test. Welch's test, $p=0.3102$. Sample size: WT, $n=13 ; \mathrm{KO}, n=13$. C, TMEM16B WT and KO littermates show no differences in latency to reach platform (left), distance traveled to reach platform (middle), and swim speed (right) during the MWM cued platform training test. Sample size: WT, $n=16$; K0, $n=13$. Error bars indicate SEM. ${ }^{* * * *} p<0.0001$.

We next sought to determine how TMEM16B KO and WT mice behave in a social setting more similar to the resident intruder paradigm. While the two-chamber social approach test measured the ability of an experimental mouse to approach the stimulus mouse, the stimulus mouse was unable to reciprocate because of its location in the enclosure. We therefore conducted the direct social interaction test, which took place in the same type of cage used during the resident intruder paradigm. During this test, the resident's home cage has been substituted with a cage unfamiliar to the resident, thereby serving as a novel environment for both the resident mouse and the social stimulus mouse. Since mice also use urine to mark their territory (Arakawa et al., 2008), removal of the home cage bedding essentially removed the sense of home cage territorial dominance of the resident mouse. Our results from this test showed no significant alterations in the amount of time TMEM16B KO mice spent interacting with the social stimulus mouse compared with WT control (Fig. 4B; Welch's $t$ test, $p=0.3102$; WT, $n=13$; KO, $n=13$ ), thereby suggesting that the increased aggression displayed by the TMEM16B KO mice is selective for the home cage environment.

Finally, we used the MWM cued platform training test to test the cohort for visual cued learning. Since TMEM16B is expressed in photoreceptors, we used this test to determine whether the observed aggression phenotype is confounded by changes in visual function in TMEM16B KO mice, and subjected the initial cohort (WT, $n=16$; KO, $n=13$ ) to the MWM cued platform training test (Fig. $4 C$ ). Based on a percentage rank analysis, TMEM16B KO mice displayed no deficits compared with WT littermates in learning to swim to the platform that served as a visual cue. TMEM16B KO mice showed no significant differences compared with WT mice in the latency to reach the platform (Fig. $4 C$, left; Welch's $t$ test, $p=0.615$ ), distance traveled to reach the platform (Fig. 4C, middle; Welch's $t$ test, $p=0.3532$ ), and swim speed (Fig. $4 C$, right; Welch's $t$ test, $p=0.7160$ ). In addition, we observed a display of visual response by every mouse in this cohort during the visual placing response, a basic neurological assessment of vision. Overall, these observations support the notion that the aggression phenotype exhibited by the TMEM16B $\mathrm{KO}$ mice during the resident intruder paradigm is due to increased displays of aggressive behaviors, and not confounding variables arising from impaired non-aggression-related social interaction or visual learning and function.

\section{Major excitatory synaptic inputs to the LS are maintained in TMEM16B KO mice}

Based on the results from the resident intruder paradigm, we reasoned that TMEM16B may contribute to the function of LS neurons involved in regulation of aggression. In the hippocampus, TMEM16B resides within the nanodomains (Augustine et al., 2003) of excitatory glutamate receptors and regulates EPSP amplitude in CA1 neurons (Huang et al., 2012). We therefore tested whether TMEM16B affects excitatory postsynaptic responses in LS neurons.

To determine whether synaptic responses of LS neurons are altered in the TMEM16B KO mice, we used optogenetics to stimulate projections from specific input regions (Fig. 5A). The LS receives inputs from a variety of brain regions associated with the limbic system (Sheehan et al., 2004). We chose to focus on two major input regions: the hippocampus and the hypothalamus (Swanson and Cowan, 1979; Staiger and Nürnberger, 1991a,b; Risold and Swanson, 1996, 1997). Based on the high mCherry expression we observe in the LSc, we chose hippocampal CA3 region and the LHA as injection sites because these brain regions innervate the LSc (Risold and Swanson, 1997). Using a stereo- 
A

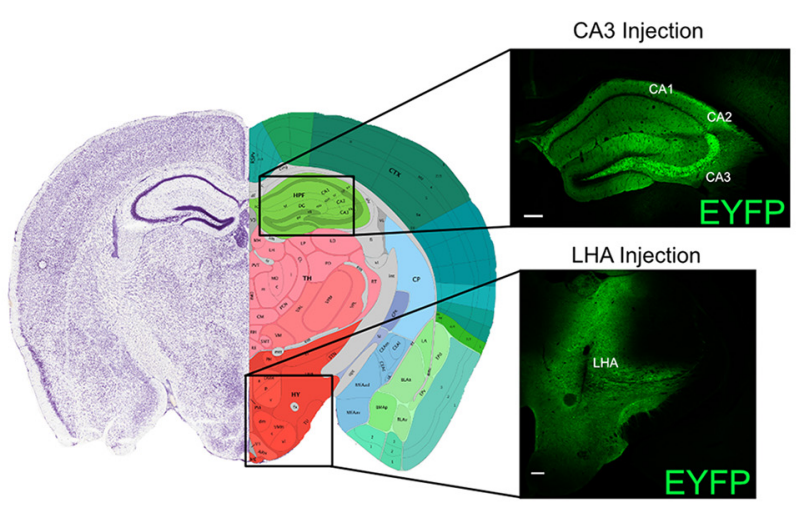

B

C
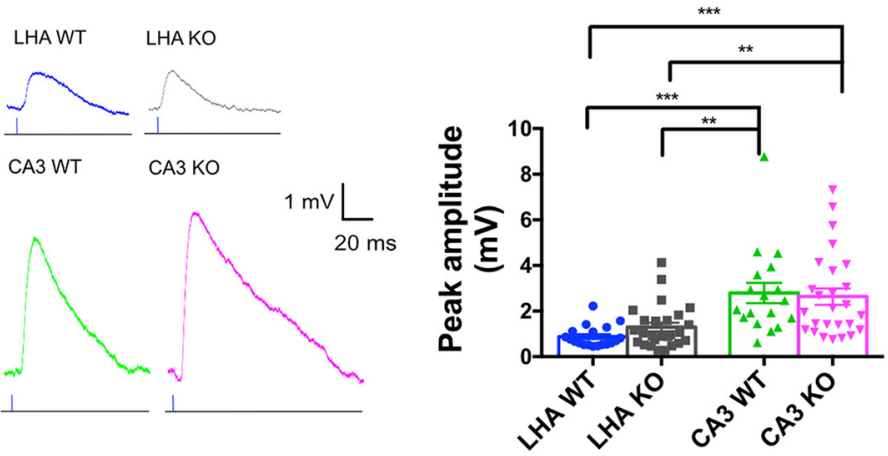

D

E
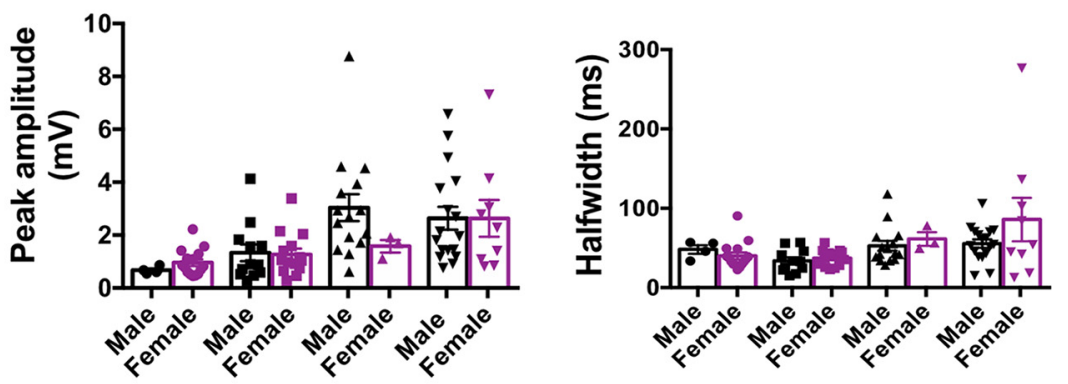

$\begin{array}{ll}\bullet & \text { LHAWT } \\ \square & \text { LHAKO } \\ \Delta & \text { CA3 WT } \\ \nabla & \text { CA3 KO }\end{array}$

F

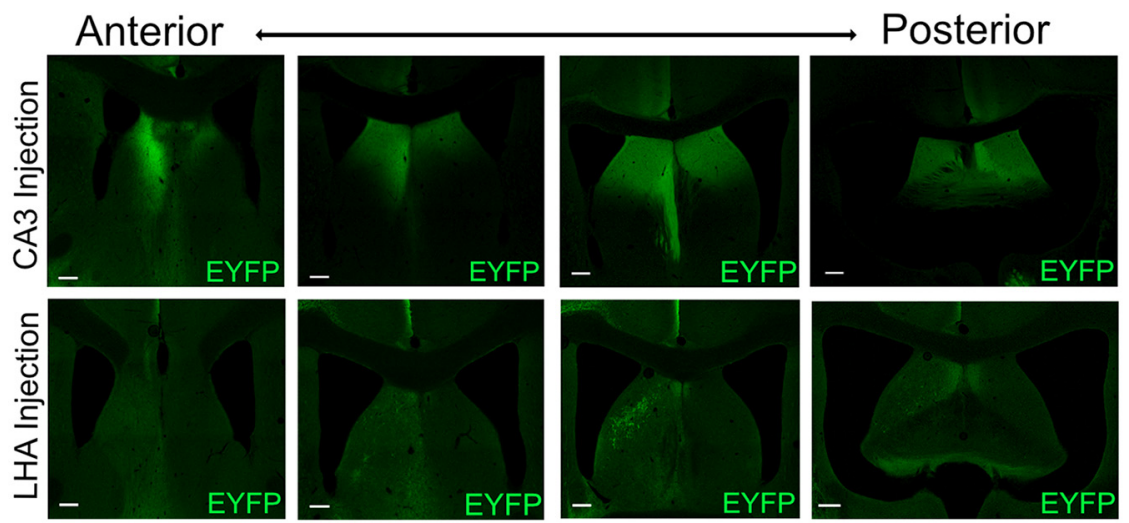

Figure 5. Major excitatory synaptic inputs to the LS are maintained in TMEM16B K0 mice. A, AAV5-CaMKII-hChR2(H134R)-EYFP was stereotaxically injected into two major excitatory LS input regions: CA3 region of the hippocampus and the LHA. Coronal image modified from Allen Brain Reference Atlas. Scale bars, $200 \mu \mathrm{m}$. $\boldsymbol{B}$, Representative traces of evoked EPSP responses from optogenetic stimulation of ChR2 in the LS; 1 ms pulse of blue light was flashed onto $L S$ neurons. $C, D, T M E M 16 B$ KO mice show no difference in evoked EPSP amplitude ( $\boldsymbol{C}$ ) or EPSP half-width (D) compared with WT, regardless of input region. For EPSP peak amplitude, CA3-evoked EPSPS show higher amplitude compared with LHA-evoked EPSPs (C). For EPSP half-width, CA3-evoked EPSPs display longer half-widths in TMEM16B K0 compared with LHA-evoked EPSPs in TMEM16B KO $(p<0.0001)$ mice (D). Two-way ANOVA with Tukey's multiple comparisons: ${ }^{* *} p<0.01 ;{ }^{* * *} p<$ 0.001. Sample size: LHA WT, $n=19 ;$ LHA KO, $n=26$; CA3 WT, $n=18 ;$ CA3 K0, $n=26$. Error bars indicate SEM. E, Comparison of male and female mice among TMEM16B WT and TMEM16B K0 genotypes shows no differences in EPSP peak amplitude (left) or half-width (right); Student's t test analysis. LHA sample size: WT female, $n=15 ; \mathrm{WT}$ male, $n=4 ; \mathrm{KO}$ female, $n=14 ; \mathrm{KO}$ male, $n=$ 12. CA3 sample size: WT female, $n=3$; WT male, $n=15 ; \mathrm{KO}$ female, $n=9 ; \mathrm{KO}$ male, $n=17$. Error bars indicate SEM. $\boldsymbol{F}$, Histological images of fixed LS coronal sections following unilateral AAV injection show expression of ChR2-EFYP in CA3 and LHA from the anterior to posterior end of the LS. Scale bars, $200 \mu \mathrm{m}$. 
A

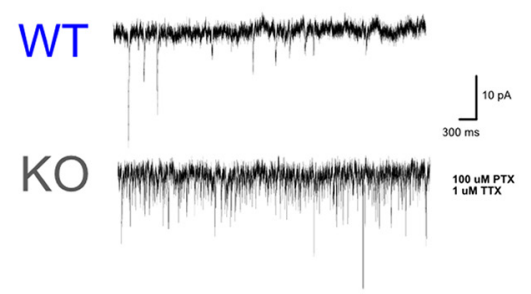

$\mathbf{E}$
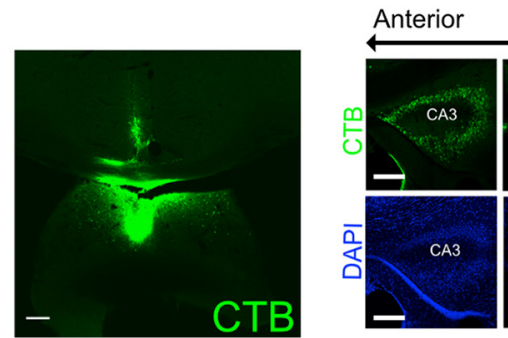

B

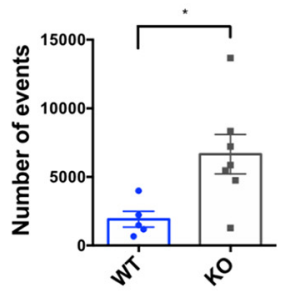

C

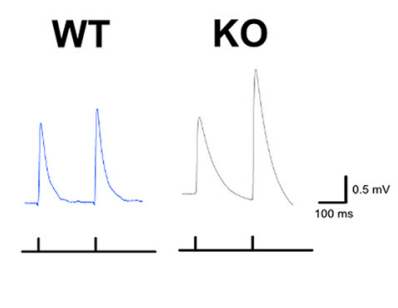

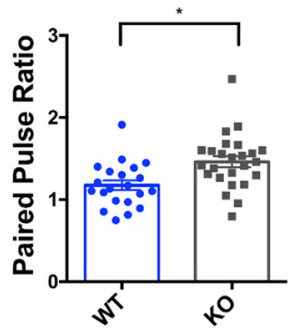

$F$
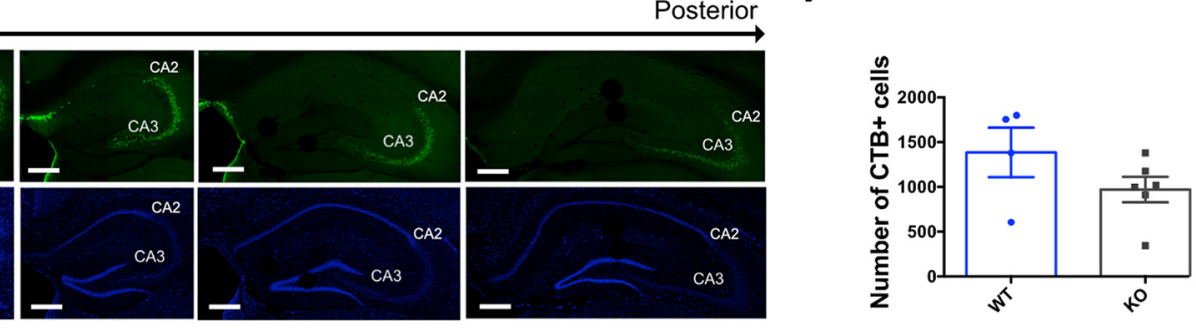

Figure 6. TMEM16B KO mice show altered presynaptic neurotransmitter release at hippocampal-LS synapse. $A$, Representative traces of voltage-clamp mEPSC recordings of TMEM16B WT and KO neurons. $\boldsymbol{B}$, Increased mEPSC frequency observed in TMEM16B KO LS neurons compared with WT. Student's $t$ test analysis: $p=0.0245$. Sample size: WT, $n=5 ; \mathrm{KO}, n=7$. C, Representative traces of EPSP paired pulse recordings using optogenetic activation of ChR2 expression in CA3. $D$, Increase in paired pulse facilitation observed in TMEM16B K0 neurons compared with WT. Student's $t$ test analysis: $p=0.0029$. Sample sizes: WT, $n=21 ; \mathrm{K} 0, n=25$. $\boldsymbol{E}$, Injection of CTB ${ }^{+}$with AlexaFluor-488 conjugation into the LSc (left) shows CTB expression in the CA2 and CA3 regions of the hippocampus along the anterior to posterior axis (right). Scale bars, $200 \mu \mathrm{m} . F$, Quantification of CTB ${ }^{+}$cells in CA3 between TMEM16B WT and K0 mice show no significant difference $(p=0.1795)$. Student's $t$ test. WT, $n=4 ; \mathrm{K} 0, n=6$. Error bars indicate SEM. ${ }^{*} p<0.05$.

taxic technique, we gave unilateral injections of the AAV5CaMKII-hChR2(H134R)-EYFP virus into either CA3 or LHA. This allowed us to express channelrhodopsin (ChR2) specifically in excitatory projection neurons that innervate LS neurons. Following stereotaxic injections, brains were sliced and prepared for whole-cell recordings in ACSF solution with $100 \mu \mathrm{M}$ PTX.

ChR2 at the axon terminals of presynaptic neurons from the input region with viral injection was activated by blue light filtered through 1-mm-thick 470/40 nm excitation filters with $1 \mathrm{~ms}$ light pulses, and the evoked EPSPs were recorded from LS neurons (Fig. 5B). When examining evoked EPSPs due to one input source alone, we found no significant differences in EPSP peak amplitude (Fig. 5C; ordinary two-way ANOVA, sources of variations: interaction, $F_{(1,85)}=0.8829, p=0.3501$; genotype, $F_{(1,85)}$ $=0.1829, p=0.6699$; brain region, $F_{(1,85)}=28.25, p<0.0001$; Tukey's multiple comparisons: WT LHA vs WT CA3, $p=0.0006$; WT LHA vs KO LHA, $p=0.7642$; WT LHA vs KO CA3, $p=$ 0.0006; WT CA3 vs KO LHA, $p=0.0051$; WT CA3 vs KO CA3, $p=0.9840$; KO LHA vs KO CA3, $p=0.0057$ ), or EPSP half-width (Fig. 5D; ordinary two-way ANOVA, sources of variations: interaction, $F_{(1,85)}=1.744, p=0.1902$; genotype, $F_{(1,85)}=0.1784, p=$ 0.6738 ; brain region, $F_{(1,85)}=9.931, p<0.0022$; Tukey's multiple comparisons: WT LHA vs WT CA3, $p=0.6302$; WT LHA vs KO LHA, $p=0.9187$; WT LHA vs KO CA3, $p=0.0599$; WT CA3 vs KO LHA, $p=0.2297$; WT CA3 vs KO CA3, $p=0.6142 ; \mathrm{KO}$ LHA vs $\mathrm{KO}$ CA3, $p=0.0045$ ) between TMEM16B KO and WT neurons. However, when we compared evoked EPSPs due to CA3 input with evoked EPSPs due to LHA input, we found that CA3evoked EPSPs showed a greater peak amplitude compared with that of LHA-evoked EPSPs (Fig. 5C). These results were consistent across both WT neurons and TMEM16B KO neurons and found to be applicable in both male and female mice (Fig. 5E; Student's $t$ test of male vs female mice; left graph, peak amplitude: LHA WT, $p=0.2695$; LHA KO, $p=0.8859$; CA3 WT, $p=$ 0.2303 ; CA3 KO, $p=0.9923$; right graph, half-width: LHA WT, $p$ $=0.3920 ; \mathrm{LHA} \mathrm{KO}, p=0.4892$; CA3 WT, $p=0.5603$; CA3 KO, $p=0.1586)$. These studies thus revealed no significant difference in the EPSPs of LS neurons in response to optogenetic stimulation of these excitatory synaptic inputs in TMEM16B KO mice compared with WT controls.

We verified the viral injection into hippocampus and LHA via immunofluorescence (Fig. 5A); CA3 as well as CA2 neurons express ChR2-EYFP and could correspond to the optogenetically activated neurons that excite LS neurons in the dorsal regions, including LSc and LSr (Leroy et al., 2018). Immunofluorescence images of ChR2-EYFP expression in the LS were also characterized (Fig. $5 F$ ), revealing bilateral and dense hippocampal projections in contrast to the unilateral and sparse projection from LHA. Overall, these results suggest that synaptic connections to the LS are maintained in TMEM16B KO mice.

\section{Loss of TMEM16B function affects neurotransmitter release} at the hippocampal-LS synapse

Because TMEM16B function has been previously identified in hippocampal pyramidal neurons, including CA3 neurons (Huang et al., 2012), we wondered whether TMEM16B could regulate neurotransmitter release at the hippocampal-LS synapse, even though we found no significant difference in the optogenetically induced EPSPs. Although no studies have identified the presence of TMEM16B at the hippocampal axon terminals thus far, TMEM16B function has been reported at the axon terminal of photoreceptors (Stöhr et al., 2009). Thus, it is plausible that TMEM16B may play a role at the hippocampal axon terminals.

Whole-cell voltage-clamp recordings of TMEM16B WT and KO LS neurons were conducted to look for differences in mEPSC firing (Fig. 6A). Cells were clamped at $-70 \mathrm{mV}$ and recorded for $10 \mathrm{~min}$. The ACSF bath solution included $1 \mu \mathrm{M}$ TTX and $100 \mu \mathrm{M}$ PTX to block action potentials and inhibitory synaptic responses, respectively. TMEM16B KO neurons showed a significant increase in mEPSC firing frequency, with $6657 \pm 1439$ events 
A

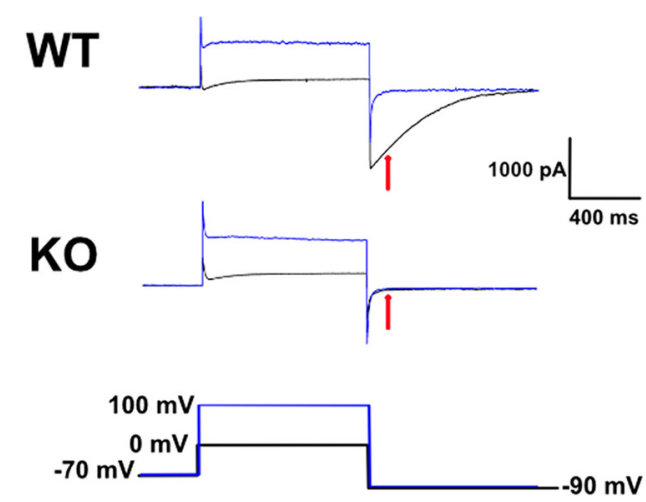

B

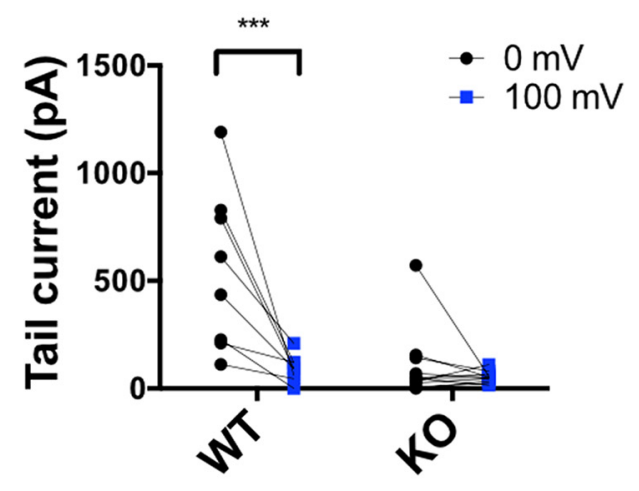

C

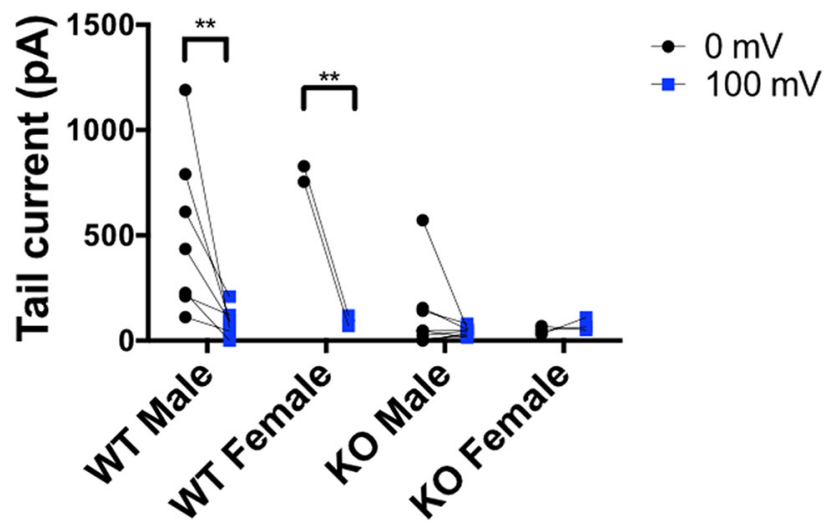

Figure 7. TMEM16B KO LS neurons show loss of CaCC. $A$, Representative traces of $\mathrm{Cl}^{-}$tail currents in WT and TMEM16B KO LS neurons. Whole-cell recordings were performed in voltage-clamp mode, and tail currents were measured $100 \mathrm{~ms}$ following a pulse to $-90 \mathrm{mV}$ (red arrow). Black traces represent pulses given from $-70 \mathrm{mV}$ to $0 \mathrm{mV}$. Blue traces represent pulses given from -70 to $100 \mathrm{mV}$. B, WT LS neurons display a tail current at $-90 \mathrm{mV}$ following an activating pulse to $0 \mathrm{mV}$ that is significantly smaller when the neuron is activated to $100 \mathrm{mV}$ instead $(p=0.0003$ ). TMEM16B KO LS neurons exhibit reduced tail current amplitudes following a $0 \mathrm{mV}$ activating pulse that are not significantly affected by preactivation at $100 \mathrm{mV}(p=0.7821)$. Repeated-measures two-way ANOVA with Sidak's multiple comparisons. Sample size: WT, $n=8 ; \mathrm{KO}, n=11$. C, Both male and female TMEM16BWT LS neurons display tail current following a $0 \mathrm{mV}$ pulse and subsequent loss of tail current following a $100 \mathrm{mV}$ pulse. Both male and female TMEM16B KO LS neurons show loss of tail current at 0 and $100 \mathrm{mV}$ pulses. Repeated-measures two-way ANOVA with Sidak's multiple comparisons. Sample size: WT male, $n=7$; WT female, $n=2 ; \mathrm{KO}$ male, $n=7 ; \mathrm{KO}$ female, $n=2$. Error bars indicate SEM. ${ }^{* *} p<0.01,{ }^{* * *} p<0.001$.

(mean \pm SEM), compared with WT neurons, with $1914 \pm 578$ events (mean \pm SEM) (Fig. $6 B$; Student's $t$ test, $p=0.0245$ ).

To look for indications of presynaptic TMEM16B function attributable to the hippocampal input, we optogenetically stimulated hippocampal CA3 projections expressing ChR2 via AAV5CaMKII-hChR2(H134R)-EYFP injection and recorded from LS neurons to quantify the paired pulse ratio (Fig. $6 \mathrm{C}$ ) as a measure of presynaptic function (Debanne et al., 1996). With a stimulation interval of $120 \mathrm{~ms}$ between pulses, EPSPs in response to optogenetic stimulation of CA3 fibers displayed paired pulse facilitation (Fig. 6D). Moreover, there was an increase in paired pulse facilitation (mean paired pulse ratio $=1.462$ ) in LS neurons from TMEM16B KO mice compared with WT controls (mean paired pulse ratio $=1.176)$, Student's $t$ test, $p=0.0029$.

To determine whether these differences were due to lack of TMEM16B channel function or changes in development due to TMEM16B deletion, we quantified the number of hippocampal-LS projections in TMEM16B KO and WT mice (Fig. $6 E, F)$. We injected the retrograde neuronal tracer, CTB conjugated to AlexaFluor-488, into the LSc and observed CTB labeling of CA3 neurons along the anterior to posterior axis of the hippocampus (Fig. 6E). Quantification of $\mathrm{CTB}^{+}$cells in CA3 showed no significant difference in the number of $\mathrm{CTB}^{+}$cells between TMEM16B WT, with a count of $1385 \pm 276$ (mean \pm SEM) $\mathrm{CTB}^{+}$cells, and TMEM16B KO, with a count of $970 \pm 142$ $\left(\right.$ mean \pm SEM) $\mathrm{CTB}^{+}$cells (Fig. $6 F$; Student's $t$ test, $p=0.1795$ ). Based on these results, we conclude that differences seen in mEPSC frequency and paired pulse facilitation are due to loss of presynaptic TMEM16B function and not differences in the number of hippocampal-LS synapses.

\section{CaCCs are present in LS neurons of WT but not TMEM16B KO mice}

To test whether TMEM16B channels are functional in LS cell bodies, we recorded calcium-activated tail currents in voltageclamp mode (Fig. 7). To isolate the contribution of CaCCs, we used ACSF where $\mathrm{NaCl}$ was replaced with NMDG-Cl, which also contained TEA-Cl and 4-AP to block $\mathrm{K}^{+}$channels. The internal solution included $\mathrm{CsCl}$ and $\mathrm{TEA}-\mathrm{Cl}$ in lieu of $\mathrm{KCl}$ to eliminate outward currents due to $\mathrm{K}^{+}$efflux. $\mathrm{Cl}^{-}$concentrations were high in both solutions to generate a theoretical $\mathrm{E}_{\mathrm{Cl}}$ of $0 \mathrm{mV}$. Cells were held at $-70 \mathrm{mV}$ and pulsed to $0 \mathrm{mV}$ for $1 \mathrm{~s}$ to allow for activation of VGCCs and $\mathrm{Ca}^{2+}$ influx (Fig. 7A, black traces). Cells were then pulsed down to $-90 \mathrm{mV}$ to elicit tail currents at a potential with a large outward driving force for $\mathrm{Cl}^{-}$to induce efflux. As a same cell negative control, cells were then pulsed from $-70 \mathrm{mV}$ to 100 
$\mathrm{mV}$ to activate $\mathrm{Ca}_{\mathrm{v}}$ channels but eliminate the inward driving force for $\mathrm{Ca}^{2+}$ ions, with the goal of reducing the intracellular concentration of $\mathrm{Ca}^{2+}$ available to activate CaCCs (Fig. 7A, blue traces). Using this protocol, we found that WT LS neurons displayed CaCC tail current of $550.9 \pm 131.9 \mathrm{pA}$ (mean \pm SEM) with a $0 \mathrm{mV}$ prepulse, which decreased significantly to $93.26 \pm$ $21.96 \mathrm{pA}$ with a $100 \mathrm{mV}$ prepulse. In contrast, TMEM16B KO LS neurons displayed a mean CaCC tail current of $103.8 \pm 49.11 \mathrm{pA}$ with a $0 \mathrm{mV}$ prepulse and $53.05 \pm 7.946 \mathrm{pA}$ with a $100 \mathrm{mV}$ prepulse (Fig. $7 B$; repeated-measures two-way ANOVA, sources of variation: interaction, $F_{(1,17)}=10.94, p=0.0042$; genotype, $F_{(1,17)}=13.80, p<0.0017$; and membrane potential, $F_{(1,25)}=$ 17.09, $p<0.0007$; Sidak's multiple comparisons: WT, $p<$ $0.0003 ; \mathrm{KO}, p=0.7821)$. Moreover, we found that both male and female TMEM16B WT mice display CaCC tail currents (Fig. 7C, left two columns; repeated-measures two-way ANOVA, sources of variation: interaction, $F_{(1,7)}=0.9587, p=0.3606$; gender, $F_{(1,7)}=0.9182, p=0.3699$; and membrane potential, $F_{(1,7)}=$ $15.86, p=0.0053$; Sidak's multiple comparisons: male WT, $p=$ 0.0024; female WT, $p=0.0068$ ), while male and female TMEM16B KO mice show loss of CaCC tail currents (Fig. 7C, right two columns; repeated-measures two-way ANOVA, sources of variation: interaction, $F_{(1,9)}=0.9259, p=0.3611$; gender, $F_{(1,9)}=0.1293, p=0.7275$; and membrane potential, $F_{(1,9)}=0.2384, p=0.6370$; Sidak's multiple comparisons: male WT, $p=0.8724$; female WT, $p=0.9997$ ). These results suggest that LS neurons express functional CaCCs that can be eliminated by deletion of TMEM16B.

\section{TMEM16B regulates spike frequency in subpopulations of LS neurons}

Once we confirmed the presence of a functional CaCC in WT LS neurons but not TMEM16B KO LS neurons, we investigated the contribution of TMEM16B to neuronal excitability in these cells. TMEM16B has previously been shown to regulate spike frequency and/or spike frequency adaptation in various brain regions, including the inferior olivary nucleus (Zhang et al., 2017), thalamacortical neurons (Ha et al., 2016), and the olfactory bulb (Pietra et al., 2016). Hence, we compared spike-firing properties between LS neurons of TMEM16B KO mice and WT controls (Fig. 8).

LS neurons were recorded in current-clamp mode and given $1 \mathrm{~s}$ current injections of increasing amplitude. The ACSF included $100 \mu \mathrm{M}$ PTX and $1 \mathrm{~mm}$ kynurenic acid to block all synaptic inputs. We found that LS neurons exhibited variability in their spike-firing characteristics, and we could functionally categorize the neurons into three easily distinguishable types, which we denoted as "tonic cells" (Fig. 8A, left; WT, $n=21 ; \mathrm{KO}, n=20$ ), and "burst cells" (Fig. 8B, left; WT, $n=13$; KO, $n=18$ ), and "phasic cells" (Fig. 8C, left; WT, $n=27$; KO, $n=25$ ). Our identification of subpopulations of LS neurons is consistent with previously published studies, which describe LS neurons with similar firing patterns (Gallagher et al., 1995; Kodirov et al., 2014). Tonic cells were characterized by regular and repetitive firing throughout the pulse duration. As current injection amplitude was elevated, tonic $\mathrm{KO}$ neurons showed increased spike frequency compared with tonic WT neurons (Fig. 8A, middle; multiple Student's $t$ test; for $p$ values, see Table 1). Tonic TMEM16B KO cells also displayed a significant increase in instantaneous frequency in the first few spikes fired during the current pulse compared with WT (Fig. $8 A$, right; multiple Student's $t$ test; for $p$ values, see Table 2), suggesting that TMEM16B plays a role in regulating the instantaneous frequency of spike firing immediately after depolariza- tion. Next, the cell type we denoted as burst cells demonstrated cyclic spike firing (Fig. 8B, left). No significant difference in spike frequency or spike frequency adaptation was detected in burst cells in TMEM16B KO versus WT (Fig. $8 B$, middle; multiple Student's $t$ test; for $p$ values, see Table 1; Fig. $8 B$, right; multiple Student's $t$ test; for $p$ values, see Table 2). Finally, phasic cells were characterized by action potential firing exclusively at the beginning of the pulse duration, followed by quiescence, which could only be relieved by removal of the current injection. However, with elevated current injection amplitude, phasic KO neurons did not show increased spike frequency compared with phasic WT neurons (Fig. 8C, middle; multiple Student's $t$ test; for $p$ values, see Table 1). These subpopulations of LS neurons were observed in both male and female WT mice, and the observed phenotypes were consistent across genders in the TMEM16B KO mice (Fig. $8 D$; multiple Student's $t$ test; for $p$ values, see Table 3 ).

We also compared the distribution of tonic, phasic, and burst cells between TMEM16B KO and WT LS neurons and observed no changes in the percentages of tonic, phasic, and burst cells (Fig. 8E). Our sample of TMEM16B WT LS neurons include $37.18 \%$ tonic cells, $41.03 \%$ phasic cells, and $21.79 \%$ burst cells, whereas our sample of TMEM16B KO LS neurons include $35.16 \%$ tonic cells, $40.66 \%$ phasic cells, and $24.18 \%$ burst cells. These findings suggest that the phenotype of TMEM16B KO mice in spike frequency and instantaneous frequency of tonic cells did not arise from a shift in the distribution of tonic cells. In conclusion, our findings suggest that TMEM16B regulates spike frequency and spike frequency adaptation for a specific subpopulation of LS neurons.

\section{Discussion}

In this study, we found that a majority of neurons in the LSc and LSr are marked by mCherry in a TMEM16B mCherry KO mouse line (Zhang et al., 2017), indicative of TMEM16B expression in these dorsal regions of LS. We investigated male aggression in mutant mice with loss of TMEM16B function, and found that TMEM16B regulates the duration of time mice spend engaged in aggressive behaviors. We found that TMEM16B function at the hippocampal axon terminal affects neurotransmitter release at the hippocampal-LS synapse. In addition, activation of voltagegated calcium channels in LS neurons resulted in a calciumactivated chloride tail current, which was eliminated in mutants with loss of TMEM16B function. Consistent with this finding of CaCC activation following calcium influx through calcium channels, we identified TMEM16B function in regulating spike frequency and spike frequency adaptation in LS neurons.

Our results suggest that the loss of the ostensibly inhibitory chloride currents through TMEM16B channels increases the neuronal excitability of subpopulation of LS neurons and increases aggressive behaviors in male mice. Our findings are in agreement with the neural circuit for regulating aggression via hippocampal CA2 innervation of the LS (Leroy et al., 2018). This recent study demonstrates that the dorsal LS, which overlaps with LS regions we refer to as the LSc and LSr (Risold and Swanson, 1997), receives excitatory input from CA2 as well as CA3; activation of dorsal LS inhibits downstream LSv neurons, thus silencing their inhibitory input to the VMH. Consequently, silencing CA2 reduces aggression in male mice during the resident intruder paradigm. Given our findings that loss of TMEM16B function alters neurotransmitter release at the hippocampal-LS synapse and increases spike firing in dorsal LS neurons, TMEM16B KO mice likely have strengthened inhibition of the LSv neuron by other LS neurons that normally express TMEM16B, leading to 
A
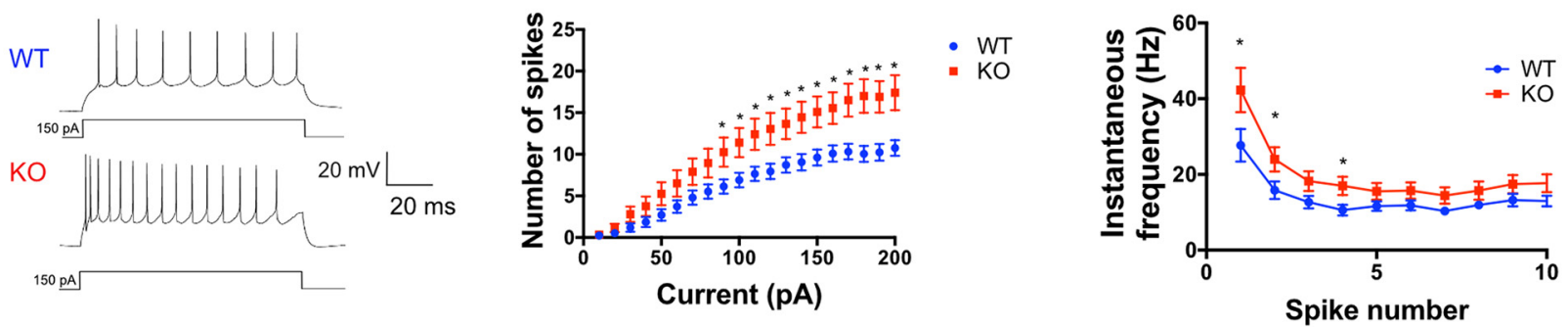

B
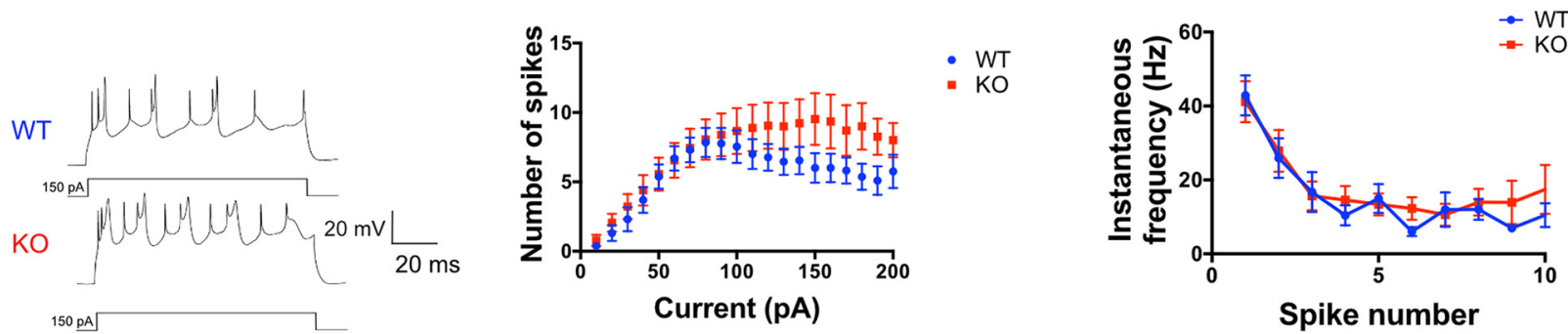

Spike number

C

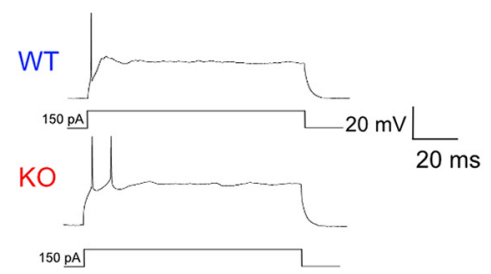

D
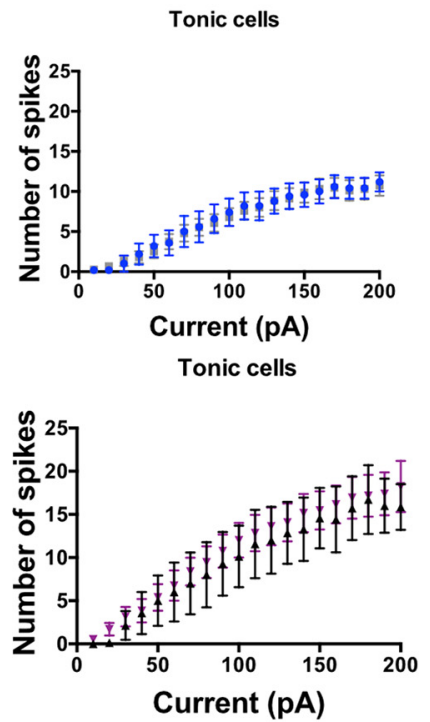

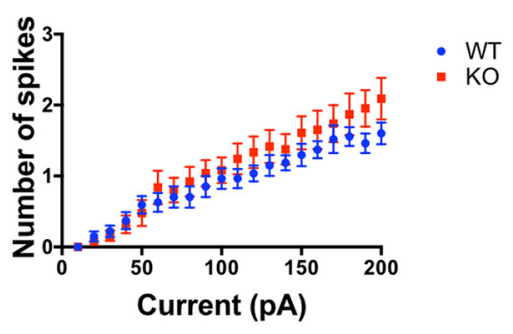

E

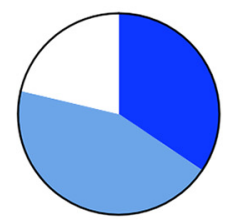

$37.18 \%$ WT Tonic $41.03 \%$ WT Phasic $21.79 \%$ WT Burst

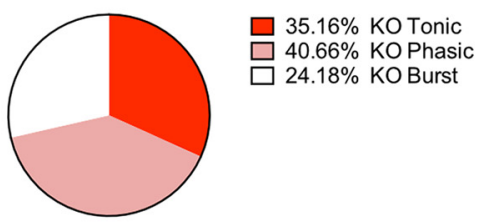

Burst cells

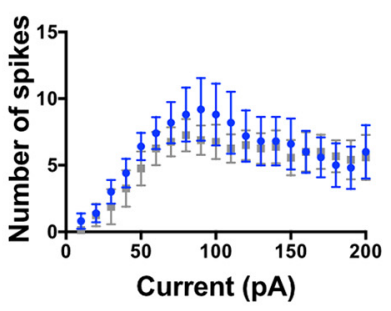

Burst cells

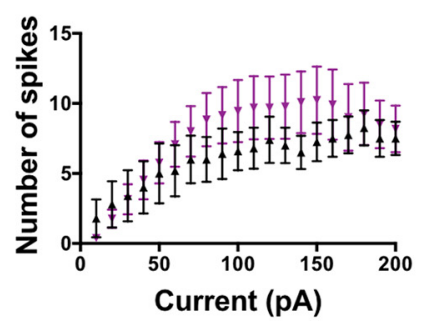

Phasic cells

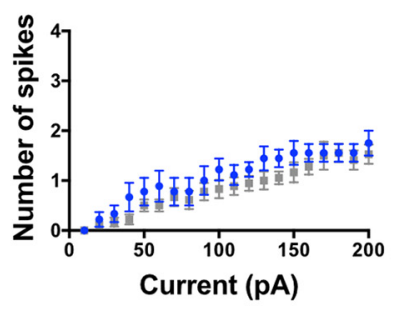

- WT Female

- WT Male

Phasic cells

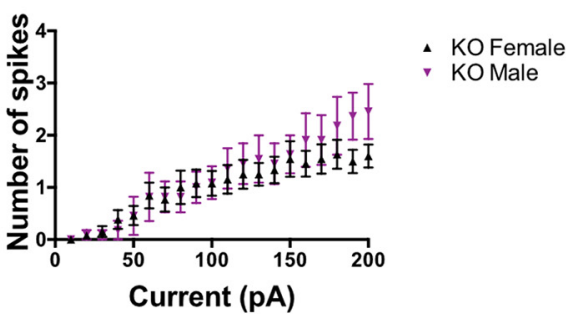

Figure 8. TMEM16B regulates action potential firing in LS neurons. $\boldsymbol{A}-\boldsymbol{C}$, Left, Representative traces of three distinct firing behaviors, denoted "tonic cells" ( $\boldsymbol{A})$, "burst cells" (B), and "phasic cells" $(C)$ are shown during 1-s-long current injections at $150 \mathrm{pA}$. Middle, Input- output curve of mean spike number. TMEM16B KO cells show significant increase in spike number compared with WT in tonic cells $(\boldsymbol{A})$, but not burst cells $(\boldsymbol{B})$ or phasic cells $(\boldsymbol{C})$. Multiple Student's t test analysis conducted; tonic cells: $p<0.05$ for current injections from 90 to $200 \mathrm{pA}$; burst and phasic cells: $p>0.05$ for all current injections. For $p$ values, see Table 1. Right, Instantaneous frequency for tonic and burst cells calculated at $150 \mathrm{pA}$ current injection. TMEM16B K0 tonic cells show increased instantaneous frequency at the first, second, and fourth spikes fired compared with WT. TMEM16B KO burst cells show no significant difference in instantaneous frequency compared with WT. Multiple Student's $t$ test (for $p$ values, see Table 2). D, Comparison of male and female TMEM16B WT and TMEM16B KO mice show no difference in spike-firing frequency. Multiple Student's $t$ test analysis (for $p$ values, see Table 3). Tonic cells sample size: male WT, $n=15$; female WT, $n=5$; male KO, $n=13$; female K0, $n=7$. Burst cells sample size: male WT, $n=8 ;$ female WT, $n=5$; male K0, $n=13 ;$ female KO, $n=5$. Phasic cells sample size: male WT, $n=18 ;$ female WT, $n=9 ;$ male K0, $n=11$; female K0, $n=13$. E, Pie chart distribution of tonic, phasic, and burst cells in WT and TMEM16B K0 mice. Sample size: tonic cells WT, $n=21 ; \mathrm{K} 0, n=20$; phasic cells WT, $n=27 ; \mathrm{K} 0, n=25$; burst cells WT, $n=13 ; \mathrm{KO}, n=18$. Error bars indicate SEM. ${ }^{*} p<0.05$. 
Table 1. Statistical analysis of spike numbers for $L S$ subpopulations of neurons ${ }^{a}$

\begin{tabular}{|c|c|c|c|c|c|}
\hline \multicolumn{2}{|l|}{ Tonic cells } & \multicolumn{2}{|l|}{ Phasic cells } & \multicolumn{2}{|l|}{ Burst cells } \\
\hline Current (pA) & $p$ & Current (pA) & $p$ & Current (pA) & $p$ \\
\hline 10 & 0.687003 & NA & NA & 10 & 0.459418 \\
\hline 20 & 0.303013 & 20 & 0.452063 & 20 & 0.408045 \\
\hline 30 & 0.12734 & 30 & 0.578307 & 30 & 0.484975 \\
\hline 40 & 0.157265 & 40 & 0.773888 & 40 & 0.650584 \\
\hline 50 & 0.104904 & 50 & 0.607294 & 50 & 0.915286 \\
\hline 60 & 0.117704 & 60 & 0.431423 & 60 & 0.934802 \\
\hline 70 & 0.0890973 & 70 & 0.674078 & 70 & 0.939876 \\
\hline 80 & 0.0790726 & 80 & 0.396248 & 80 & 0.914621 \\
\hline $90^{*}$ & 0.0393411 & 90 & 0.430464 & 90 & 0.765176 \\
\hline $100^{*}$ & 0.0247796 & 100 & 0.615486 & 100 & 0.61091 \\
\hline $110^{*}$ & 0.0262709 & 110 & 0.278638 & 110 & 0.394572 \\
\hline $120^{*}$ & 0.0195994 & 120 & 0.225632 & 120 & 0.291496 \\
\hline $130^{*}$ & 0.0196844 & 130 & 0.324088 & 130 & 0.248996 \\
\hline $140^{*}$ & 0.0139169 & 140 & 0.418522 & 140 & 0.223559 \\
\hline $150^{*}$ & 0.0112489 & 150 & 0.261719 & 150 & 0.153725 \\
\hline $160^{*}$ & 0.0133945 & 160 & 0.323187 & 160 & 0.201719 \\
\hline $170^{* *}$ & 0.00693941 & 170 & 0.494336 & 170 & 0.240875 \\
\hline $180^{* *}$ & 0.00320534 & 180 & 0.316105 & 180 & 0.108232 \\
\hline $190^{* *}$ & 0.00322645 & 190 & 0.0864734 & 190 & 0.100398 \\
\hline $200^{* *}$ & 0.00576751 & 200 & 0.131641 & 200 & 0.255281 \\
\hline
\end{tabular}

${ }^{a}$ The $p$ values for multiple Student's $t$ test analysis of spike numbers at incremental current injections for tonic cells, phasic cells, and burst cells in the LS.

${ }^{*} p<0.05$, ** $p<0.01$.

Table 2. Statistical analysis of instantaneous frequency for LS subpopulations of neurons $^{a}$

\begin{tabular}{lllll}
\hline Tonic cells & & & \multicolumn{2}{l}{ Burst cells } \\
\cline { 5 - 5 } Spike number & $p$ & & Spike number & $p$ \\
\hline $1^{*}$ & 0.0492253 & 1 & 0.836921 \\
$2^{*}$ & 0.0434869 & 2 & 0.820201 \\
3 & 0.0774943 & 3 & 0.878783 \\
$4^{*}$ & 0.0259649 & 4 & 0.468033 \\
5 & 0.132263 & 5 & 0.774184 \\
6 & 0.143233 & 6 & 0.286683 \\
7 & 0.110031 & 7 & 0.808547 \\
8 & 0.18395 & 8 & 0.809417 \\
9 & 0.205672 & 9 & 0.510189 \\
10 & 0.147416 & 10 & 0.529261
\end{tabular}

${ }^{a}$ The $p$ values for multiple Student's $t$ test analysis of instantaneous frequency at specific spikes fired during the current injection for tonic cells and burst cells.

${ }^{*} p<0.05$. disinhibition of the VMH and an increase in aggressive behaviors as we observed.

In addition to the role of the hippocampus as a mediator of LS-induced aggression, the hallmark functions of the hippocampus in episodic memory (Tulving and Markowitsch, 1998) and cognitive map formation (O'Keefe and Nadel, 1978) may also contribute to aggression, especially given the setup of our resident intruder paradigm. The ability of the resident mouse to recognize his home cage is reflective of his ability to form a cognitive map of his environment. Moreover, the repeated exposure to an intruder mouse requires the formation of a specific type of episodic memory, social memory, or the ability of an individual to remember someone from the same species. The CA2 region of the hippocampus is known to be essential for mediating social memory recognition (Hitti and Siegelbaum, 2014; Stevenson and Caldwell, 2014), and CA3 has been found to be a contributor as well (Chiang et al., 2018). Conceivably, the increased attack times displayed by the TMEM16B KO mice could be due to an enhanced ability for social memory recall of the intruder mice that elicits an increase in territorial aggression.

Our findings provide evidence that activation of dorsal regions of the LS promotes aggression (Leroy et al., 2018). Although the classical understanding attributes LS function to suppressing aggression, many of these studies involved broadly manipulating the LS, via lesions (Spiegel et al., 1940; Brady and Nauta, 1953; Albert and Richmond, 1976), electrical stimulation (Brayley and Albert, 1977), or optogenetic activation (Wong et al., 2016). Thus, these studies do not take into account the complex LS microcircuitry that has only begun to be elucidated (Leroy et al., 2018). Connections between LS subregions are known to be organized in such a way that LS neurons within an individual subregion maintain connections with one another, while also projecting in dorsoventral and mediolateral directions to neighboring LS subregions (Alonso and Frotscher, 1989; Staiger and Nürnberger, 1991b; Risold and Swanson, 1997). Further investigation of the mechanisms underlying LS microcircuit function is necessary to better understand its regulation of aggression.

While our study supports the notion that TMEM16Bexpressing LS neurons in dorsal regions are excited by hippocam-

Table 3. Statistical analysis of male versus female spike numbers for LS neuronal subpopulations ${ }^{a}$

\begin{tabular}{|c|c|c|c|c|c|c|}
\hline \multirow[b]{2}{*}{ Current (pA) } & \multicolumn{2}{|l|}{ Tonic cells } & \multicolumn{2}{|l|}{ Phasic cells } & \multicolumn{2}{|l|}{ Burst cells } \\
\hline & WT & KO & WT & KO & WT & KO \\
\hline 10 & 0.891705 & 0.197016 & NA & NA & 0.184038 & 0.123869 \\
\hline 30 & 0.79068 & 0.613339 & 0.345139 & 0.659414 & 0.550404 & 0.907069 \\
\hline 40 & 0.793633 & 0.915465 & 0.0834013 & 0.439917 & 0.567279 & 0.834892 \\
\hline 50 & 0.700605 & 0.899134 & 0.293585 & 0.985863 & 0.385251 & 0.782149 \\
\hline 60 & 0.944191 & 0.825677 & 0.17047 & 0.956262 & 0.548316 & 0.518844 \\
\hline 90 & 0.808128 & 0.697297 & 0.490305 & 0.849087 & 0.334609 & 0.4407 \\
\hline 100 & 0.784572 & 0.627481 & 0.215632 & 0.971641 & 0.41959 & 0.455411 \\
\hline 110 & 0.784172 & 0.757274 & 0.451597 & 0.65569 & 0.405895 & 0.456922 \\
\hline 120 & 0.93164 & 0.698455 & 0.254261 & 0.669903 & 0.741411 & 0.552836 \\
\hline 130 & 0.976784 & 0.76173 & 0.161329 & 0.553258 & 0.793482 & 0.480291 \\
\hline 140 & 0.913435 & 0.64766 & 0.0873779 & 0.794303 & 0.845049 & 0.398111 \\
\hline 190 & 0.978679 & 0.739518 & 0.631059 & 0.113999 & 0.788981 & 0.751892 \\
\hline 200 & 0.844603 & 0.604598 & 0.512178 & 0.166593 & 0.886521 & 0.818323 \\
\hline
\end{tabular}

${ }^{a}$ The $p$ values for multiple Student's $t$ test analysis of spike numbers in male versus female $L S$ neurons at incremental current injections for $L S$ tonic cells, phasic cells, and burst cells. 
pal neurons to cause disinhibition of VMH leading to aggressive behaviors, it remains possible that additional hypothalamic nuclei are involved in regulating aggression. We identified mCherry expression of LS axon terminals in downstream hypothalamic regions, namely, the LHA and LPO (Fig. 2). These results are consistent with LS connectivity studies revealing innervation of the LHA and LPO by both the LSc and LSr (Risold and Swanson, 1997). Notably, electrical stimulation of the LHA leads to increased aggressive behavior (Smith et al., 1970; Woodworth, 1971; Koolhaas, 1978; Kruk et al., 1983), and reciprocal connections exist between the VMH and LHA (Canteras et al., 1994). Moreover, the LPO maintains reciprocal connections with the medial preoptic area, another hypothalamic nucleus implicated in aggression (Chiba and Murata, 1985; Albert et al., 1986). Therefore, LS input to the LHA and LPO may contribute to regulating aggression as well.

Whereas the aggression phenotype of TMEM16B KO mice appears mild compared with published studies that used the resident-intruder paradigm, it is important to compare the protocols used in these studies. It is common for researchers using the resident intruder paradigm to house the "resident" male mouse with a sterile female mouse for weeks and then remove the female just before the introduction of the "intruder" mouse (Veenema et al., 2010; Koolhaas et al., 2013), to enhance the sense of territoriality of the resident mouse (Albert et al., 1988). In contrast, our study was performed with single housing of sexually naive resident mice for weeks before introducing the intruder mouse. In addition, many resident intruder paradigms only involve $1 \mathrm{~d}$ of testing, whereas ours included $3 \mathrm{~d}$ of testing. We chose this approach because repeated exposure to an intruder mouse may be required to accurately determine the aggression phenotype (Winslow and Miczek, 1983; Ogawa et al., 1998; Miczek et al., 2001). With these considerations, it is not surprising that our experiments elicited a milder intruder response, with the most pronounced differences in aggression displayed on days 2 and 3 of the test. Aggression is not an all-or-none response, and our findings regarding the aggression phenotype in TMEM16B KO mice are consistent with the general understanding that natural variability exists in behavior, even among animals with the same genotype.

Future study of the brain regions that receive innervation of TMEM16B-expression LS neurons, whether it be the LSv or downstream hypothalamic nuclei, would be instrumental in understanding how LS neurons are integrated within the broader neuronal circuitry underlying aggressive social behaviors. It seems likely that the change in spike frequency and spike frequency adaptation detected in subpopulations of LS neurons lacking TMEM16B would correlate with changes in postsynaptic activity in downstream neurons. Moreover, examination of the specific projections of the TMEM16B-expressing LS neurons may provide additional insight regarding the circuits involved in regulating aggressive behaviors.

In addition, it would be intriguing to investigate TMEM16B function in relation to LS-mediated female social behaviors. LS function is known to be involved in regulating multiple aspects of female behavior, including maternal care and aggression (Flannelly et al., 1986; D'Anna and Gammie, 2009; Lee and Gammie, 2009; Scotti et al., 2011) and female sexual behavior (Yamanouchi and Arai, 1990). While our study identified TMEM16B expression and function in female LS neurons (Figs. $1 F, 7 C, 8 D$ ), the absence of behavioral tests for the role of TMEM16B in regulating female social behaviors is a limitation of this study. Future investigations regarding how TMEM16B Ca ${ }^{2+}$-activated $\mathrm{Cl}^{-}$ channel function affects female social behaviors would provide important insight into the contribution of $\mathrm{CaCC}$ to the modulation of these behaviors.

In conclusion, our study of TMEM16B in the LS provides the first demonstration of the presence and function of CaCCs in this brain region. Our investigation of the role of TMEM16B at the physiological and behavior levels provides insight into how TMEM16B regulates LS neuronal excitability. Our study raises intriguing questions regarding how LS function and regulation may contribute to aggressive behavior. It also provides an example as to how different subregions of the LS in this complex neural circuit may have different channel compositions for activity modulation and vary in their contributions to the regulation of aggression and related behaviors.

\section{References}

Albert DJ, Chew GL (1980) The septal forebrain and the inhibitory modulation of attack and defense in the rat: a review. Behav Neural Biol 30:357-388.

Albert DJ, Richmond SE (1976) Hyperreactivity and aggressiveness following infusion of local anesthetic into the lateral septum or surrounding structures. Behav Biol 18:211-226.

Albert DJ, Wong RC (1978) Hyperreactivity, muricide, and intraspecific aggression in the rat produced by infusion of local anesthetic into the lateral septum or surrounding areas. J Comp Physiol Psychol 92:1062-1073.

Albert DJ, Walsh ML, Gorzalka BB, Mendelson S, Zalys C (1986) Intermale social aggression: suppression by medial preoptic area lesions. Physiol Behav 38:169-173.

Albert DJ, Dyson EM, Walsh ML, Petrovic DM (1988) Cohabitation with a female activates testosterone-dependent social aggression in male rats independently of changes in serum testosterone concentration. Physiol Behav 44:735-740.

Alonso JR, Frotscher M (1989) Organization of the septal region in the rat brain: a Golgi/EM study of lateral septal neurons. J Comp Neurol 286: $472-487$.

Arakawa H, Blanchard DC, Arakawa K, Dunlap C, Blanchard RJ (2008) Scent marking behavior as an odorant communication in mice. Neurosci Biobehav Rev 32:1236-1248.

Augustine GJ, Santamaria F, Tanaka K (2003) Local calcium signaling in neurons. Neuron 40:331-346.

Brady JV, Nauta WJ (1953) Subcortical mechanisms in emotional behavior: affective changes following septal forebrain lesions in the albino rat. J Comp Physiol Psychol 46:339-346.

Brady JV, Nauta WJ (1955) Subcortical mechanisms in emotional behavior: the duration of affective changes following septal and habenular lesions in the albino rat. J Comp Physiol Psychol 48:412-420.

Brayley KN, Albert DJ (1977) Suppression of VMH-lesion induced reactivity and aggressiveness by electrical stimulation ventral to the anterior septum in the rat. Physiol Behav 18:567-571.

Canteras NS, Simerly RB, Swanson LW (1994) Organization of projections from the ventromedial nucleus of the hypothalamus: a phaseolus vulgaris-leucoagglutinin study in the rat. J Comp Neurol 348:41-79.

Chiang MC, Huang AJ, Wintzer ME, Ohshima T, McHugh TJ (2018) A role for CA3 in social recognition memory. Behav Brain Res 354:22-30.

Chiba T, Murata Y (1985) Afferent and efferent connections of the medial preoptic area in the rat: a WGA-HRP study. Brain Res Bull 14:261-272.

D'Anna KL, Gammie SC (2009) Activation of corticotropin-releasing factor receptor 2 in lateral septum negatively regulates maternal defense. Behav Neurosci 123:356-368.

Debanne D, Guérineau NC, Gähwiler BH, Thompson SM (1996) Pairedpulse facilitation and depression at unitary synapses in rat hippocampus: quantal fluctuation affects subsequent release. J Physiol 491:163-176.

Flannelly KJ, Kemble ED, Blanchard DC, Blanchard RJ (1986) Effects of septal-forebrain lesions on maternal aggression and maternal care. Behav Neural Biol 45:17-30.

Gallagher JP, Zheng F, Hasuo H, Shinnick-Gallagher P (1995) Activities of neurons within the rat dorsolateral septal nucleus (DLSN). Prog Neurobiol 45:373-395.

Ha GE, Lee J, Kwak H, Song K, Kwon J, Jung SY, Hong J, Chang GE, Hwang EM, Shin HS, Lee CJ, Cheong E (2016) The Ca. Nat Commun 7:13791. 
Hitti FL, Siegelbaum SA (2014) The hippocampal CA2 region is essential for social memory. Nature 508:88-92.

Huang WC, Xiao S, Huang F, Harfe BD, Jan YN, Jan LY (2012) Calciumactivated chloride channels (CaCCs) regulate action potential and synaptic response in hippocampal neurons. Neuron 74:179-192.

Kodirov SA, Wehrmeister M, Colom LV (2014) Modulation of HCN channels in lateral septum by nicotine. Neuropharmacology 81:274-282.

Koolhaas JM (1978) Hypothalamically induced intraspecific aggressive behaviour in the rat. Exp Brain Res 32:365-375.

Koolhaas JM, Coppens CM, de Boer SF, Buwalda B, Meerlo P, Timmermans PJ (2013) The resident-intruder paradigm: a standardized test for aggression, violence and social stress. J Vis Exp 77:e4367.

Kruk MR, Van der Poel AM, Meelis W, Hermans J, Mostert PG, Mos J, Lohman AH (1983) Discriminant analysis of the localization of aggression-inducing electrode placements in the hypothalamus of male rats. Brain Res 260:61-79.

Lee G, Gammie SC (2009) GABA(A) receptor signaling in the lateral septum regulates maternal aggression in mice. Behav Neurosci 123:1169-1177.

Leroy F, Park J, Asok A, Brann DH, Meira T, Boyle LM, Buss EW, Kandel ER, Siegelbaum SA (2018) A circuit from hippocampal CA2 to lateral septum disinhibits social aggression. Nature 564:213-218.

Miczek KA, Maxson SC, Fish EW, Faccidomo S (2001) Aggressive behavioral phenotypes in mice. Behav Brain Res 125:167-181.

Ogawa S, Washburn TF, Taylor J, Lubahn DB, Korach KS, Pfaff DW (1998) Modifications of testosterone-dependent behaviors by estrogen receptoralpha gene disruption in male mice. Endocrinology 139:5058-5069.

O'Keefe J, Nadel L (1978) The hippocampus as a cognitive map. New York: Oxford UP.

Pietra G, Dibattista M, Menini A, Reisert J, Boccaccio A (2016) The $\mathrm{Ca}^{2+}$. activated $\mathrm{Cl}^{-}$channel TMEM16B regulates action potential firing and axonal targeting in olfactory sensory neurons. J Gen Physiol 148:293-311.

Pifferi S, Dibattista M, Menini A (2009) TMEM16B induces chloride currents activated by calcium in mammalian cells. Pflugers Arch 458: 1023-1038.

Risold PY, Swanson LW (1996) Structural evidence for functional domains in the rat hippocampus. Science 272:1484-1486.

Risold PY, Swanson LW (1997) Connections of the rat lateral septal complex. Brain Res Brain Res Rev 24:115-195.

Sagheddu C, Boccaccio A, Dibattista M, Montani G, Tirindelli R, Menini A (2010) Calcium concentration jumps reveal dynamic ion selectivity of calcium-activated chloride currents in mouse olfactory sensory neurons and TMEM16b-transfected HEK 293T cells. J Physiol 588:4189-4204.

Scotti MA, Lee G, Gammie SC (2011) Maternal defense is modulated by beta adrenergic receptors in lateral septum in mice. Behav Neurosci 125:434-445.

Sheehan TP, Chambers RA, Russell DS (2004) Regulation of affect by the lateral septum: implications for neuropsychiatry. Brain Res Brain Res Rev 46:71-117.

Smith DE, King MB, Hoebel BG (1970) Lateral hypothalamic control of killing: evidence for a cholinoceptive mechanism. Science 167:900-901.

Spiegel EA, Miller HR, Oppenheimer MJ (1940) Forebrain and rage reactions. J Neurophysiol 3:538-548.

Staiger JF, Nürnberger F (1991a) The efferent connections of the lateral septal nucleus in the guinea pig: projections to the diencephalon and brainstem. Cell Tissue Res 264:391-413.

Staiger JF, Nürnberger F (1991b) The efferent connections of the lateral septal nucleus in the guinea pig: intrinsic connectivity of the septum and projections to other telencephalic areas. Cell Tissue Res 264:415-426.

Stephan AB, Shum EY, Hirsh S, Cygnar KD, Reisert J, Zhao H (2009) ANO2 is the cilial calcium-activated chloride channel that may mediate olfactory amplification. Proc Natl Acad Sci U S A 106:11776-11781.

Stevenson EL, Caldwell HK (2014) Lesions to the CA2 region of the hippocampus impair social memory in mice. Eur J Neurosci 40:3294-3301.

Stöhr H, Heisig JB, Benz PM, Schöberl S, Milenkovic VM, Strauss O, Aartsen WM, Wijnholds J, Weber BH, Schulz HL (2009) TMEM16B, a novel protein with calcium-dependent chloride channel activity, associates with a presynaptic protein complex in photoreceptor terminals. J Neurosci 29:6809-6818.

Swanson LW, Cowan WM (1979) The connections of the septal region in the rat. J Comp Neurol 186:621-655.

Toth M, Fuzesi T, Halasz J, Tulogdi A, Haller J (2010) Neural inputs of the hypothalamic "aggression area" in the rat. Behav Brain Res 215:7-20.

Tulving E, Markowitsch HJ (1998) Episodic and declarative memory: role of the hippocampus. Hippocampus 8:198-204.

Veenema AH, Beiderbeck DI, Lukas M, Neumann ID (2010) Distinct correlations of vasopressin release within the lateral septum and the bed nucleus of the stria terminalis with the display of intermale aggression. Horm Behav 58:273-281.

Winslow JT, Miczek KA (1983) Habituation of aggression in mice: pharmacological evidence of catecholaminergic and serotonergic mediation. Psychopharmacology (Berl) 81:286-291.

Wong LC, Wang L, D'Amour JA, Yumita T, Chen G, Yamaguchi T, Chang BC, Bernstein H, You X, Feng JE, Froemke RC, Lin D (2016) Effective modulation of male aggression through lateral septum to medial hypothalamus projection. Curr Biol 26:593-604.

Woodworth CH (1971) Attack elicited in rats by electrical stimulation of the lateral hypothalamus. Physiol Behav 6:345-353.

Yamanouchi K, Arai Y (1990) The septum as origin of a lordosis-inhibiting influence in female rats: effect of neural transection. Physiol Behav 48:351-355.

Zhang Y, Zhang Z, Xiao S, Tien J, Le S, Le T, Jan LY, Yang H (2017) Inferior olivary TMEM16B mediates cerebellar motor learning. Neuron 95:11031111.e4. 